Portland State University

PDXScholar

Summer 1-1-2012

\title{
Measurement and modeling of passive surface mount devices on FR4 substrates
}

Rahulkumar Sadanand Koche

Portland State University

Follow this and additional works at: https://pdxscholar.library.pdx.edu/open_access_etds

Part of the Digital Circuits Commons, Electrical and Electronics Commons, and the Other Computer Sciences Commons

Let us know how access to this document benefits you.

\section{Recommended Citation}

Koche, Rahulkumar Sadanand, "Measurement and modeling of passive surface mount devices on FR4 substrates" (2012). Dissertations and Theses. Paper 754.

https://doi.org/10.15760/etd.754

This Thesis is brought to you for free and open access. It has been accepted for inclusion in Dissertations and Theses by an authorized administrator of PDXScholar. Please contact us if we can make this document more accessible: pdxscholar@pdx.edu. 
Measurement and modeling of passive surface mount devices on FR4 substrates

by

Rahulkumar Sadanand Koche

A thesis submitted in partial fulfillment of the

requirements for the degree of

\author{
Master of Science \\ in \\ Electrical and Computer Engineering
}

Thesis Committee:

Branimir Pejcinovic, Chair

Garrison Greenwood

James Morris

Portland State University

2012 


\begin{abstract}
Passive components like resistors, capacitors and inductors are used in every electronic system. These are the very basic components which affect the system performance at higher frequencies and it is necessary to understand and model the behavior of these components in a very accurate manner.

This work focuses on utilizing printed circuit board (PCB) test boards, or fixtures, made of FR4 for characterizing Surface Mount Device (SMD) components. Agilent's Advanced Design System (ADS) microwave circuit simulation software was used for designing the microstrip transmission lines as well as for generating the layout for manufacturing of the PCB. SMD resistors, capacitors and inductors were soldered into the fixture and then measured using the Vector Network Analyzer (VNA).
\end{abstract}

The calibration kit was developed in ADS. The measured data were calibrated using the TRL (Thru-Reflect-Line) calibration algorithm. A calibration kit consisting of through, three transmission lines of various lengths, open and short was designed and manufactured. Calibration procedures were performed using Cascade Microtech's WinCal XE software. Based on our experience, TRL calibration did not perform to its full potential due to errors in the value of the characteristic impedance of microstrip transmission line. This impedance is ideally assumed to be $50 \Omega$, but our lines had characteristic impedance of around $49 \Omega$.

Simple models for the resistors and capacitors were developed by our collaborators at the University of Zagreb and we developed the model for the inductors. We used ADS for simulations and comparison with the measured data. Extensive optimization of these models was done so as to fit the measured and modeled data. As the frequency goes above $4 \mathrm{GHz}$ models and measurements don't match due 
to the limitations of the PCB material, the increasing effects of the parasitics and calibration artifacts.

This work shows how and when we can use inexpensive FR4 PCB for the characterization of the passive SMD components in the low $\mathrm{GHz}$ frequency range. It also examines the range of operating frequency of SMD components, verifies the parameters extracted from the simple model and tests the TRL calibration algorithm. 


\section{Acknowledgements}

This work would not have been possible without the unending help and motivation by my advisor Dr. Branimir Pejcinovic. I would like to give him special thanks for supporting me at all times. I would like to thank the committee members Dr. Garrison Greenwood and Dr. James Morris who were present for the defense. I would like to extend a heart felt thanks to Alex who has been around and giving me useful feedback from his experiences.

Outside my school there are people who have helped me indirectly and kept me motivated always. I would like to thank my family and friends without whom this work would be incomplete. I would like to thank my family - mom, dad and Mukul for understanding me and keeping me motivated till the end of my thesis

work. A special thanks to one of my dearest friend Pallavi for being with me at all times, without your support this would not have been possible.

Last but not the least, I would like to thank my buddies - Srinivas, Vinaya, Rasika, Uttara, Prachi and Suvarna who have missed me many times for lunches and dinners. Also, I would like to thank Diane for helping me with my thesis work during my internship period. Without you all this would not have been possible.

Lastly, I would like to thank all the people who have helped me directly and/or indirectly all through my journey towards the end of my thesis

Thank you all!

Best Regards, Rahul 


\section{Contents}

Abstract $\quad$ i

$\begin{array}{ll}\text { Acknowledgements } & \text { iii }\end{array}$

List of Tables viii

List of Figures $\quad x$

1 Introduction 1

1.1 Introduction . . . . . . . . . . . . . . . . . . . . 1

1.2 Motivation .......................... 2

1.3 Need for calibration . . . . . . . . . . . . . . . . . . 3

1.4 Accurate modeling of SMD devices - Necessity . . . . . . . . . . . . 4

1.5 Thesis contribution ................... 4

1.6 Thesis layout . . . . . . . . . . . . . . . . . . 5

2 Background and Theory $\quad 6$

2.1 Scattering Parameters $[\mathrm{S}$-parameters $] \ldots \ldots \ldots$. . . . . . . . 6

2.2 Transmission Lines . . . . . . . . . . . . . . . . . 6

2.3 Definition of S-parameters . . . . . . . . . . . . . . . 7

2.3.1 S-parameter equations . . . . . . . . . . . . . 9

2.4 Impedance Measurement Basics . . . . . . . . . . . . . . . . . . . . 10

2.4.1 Ideal, real and measured values . . . . . . . . . . . . 12

2.5 Parameters that affect the measurement system . . . . . . . . . 15 
2.5.1 Test System Errors . . . . . . . . . . . . . . . . . 15

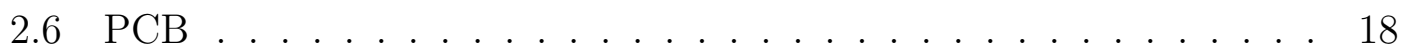

2.7 Resonator structure . . . . . . . . . . . . . . . . . . 18

2.8 Reference $\Pi$ Model . . . . . . . . . . . . . . . . . . . . . . . . . . 21

2.9 Resistor - Simple Models . . . . . . . . . . . . . . . . . . . . . 24

2.10 Capacitor - Simple Models . . . . . . . . . . . . . . . 25

2.11 Inductor - Simple Models . . . . . . . . . . . . . . . . . . 26

3 Calibration Methodology 29

3.1 Introduction to Calibration $\ldots \ldots \ldots \ldots \ldots$

3.2 Reference Plane . . . . . . . . . . . . . . . . . . . . . . 29

3.3 Calibration Standards . . . . . . . . . . . . . . 30

3.3 .1 Short . . . . . . . . . . . . . . . . . . 31

3.3 .2 Open . . . . . . . . . . . . . . . . . 31

3.3 .3 Load . . . . . . . . . . . . . . . . . . . 32

3.3 .4 THRU . . . . . . . . . . . . . . . . . 32

3.4 Basic calibration process $\ldots \ldots \ldots \ldots \ldots \ldots$

3.5 TRL (Thru-Reflect-Line) Calibration . . . . . . . . . . . 33

3.5 .1 THRU . . . . . . . . . . . . . . . . . 33

3.5 .2 Reflect . . . . . . . . . . . . . 34

3.5 .3 Line . . . . . . . . . . . . . . . . . 34

3.6 TRL line design . . . . . . . . . . . . . . . . . . . . . 35

3.7 Selecting the optimal THRU and Line lengths . . . . . . . . . 35

3.8 Cascade Microtech's WinCal XE calibration process . . . . . . . . . 39

4 Measurement and Modeling $\quad 44$ 
4.1 Measurement Setup . . . . . . . . . . . . . . . . 44

4.2 Model extraction setup - Optimization description . . . . . . . . . 47

4.2.1 Random Optimizer . . . . . . . . . . . . . . . 50

4.2 .2 Simulated Annealing . . . . . . . . . . . . . 50

4.2 .3 Gradient Optimizer ................ 51

4.3 Optimization Description . . . . . . . . . . . . . 51

4.4 Component Selection . . . . . . . . . . . . . . . . . 52

4.5 Resistor Modeling . . . . . . . . . . . . . . . . . . 52

4.6 Capacitor Modeling . . . . . . . . . . . . . . . . . 54

4.7 Inductor Modeling . . . . . . . . . . . . . . . . 54

4.8 Measurement Observations . . . . . . . . . . . . . . . . 58

5 Summary of Results and Discussion 61

5.1 Comparison of measured and modeled data - Resistor, Capacitor and Inductor . . . . . . . . . . . . . . . . . . . 6 61

5.2 Resistor Results . . . . . . . . . . . . . . . . 61

5.3 Inductor Results .................... 65

5.4 Capacitor Results ..................... 69

6 Conclusion and Future work $\quad 72$

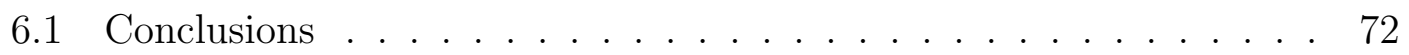

6.2 Future work .......................... 73

$\begin{array}{ll}\text { References } & 74\end{array}$

$\begin{array}{ll}\text { A Plots } & 76\end{array}$

A.1 Resistor plots .................... 76 
A.2 Capacitor plots .................... . . 76

A.3 Inductor plots . . . . . . . . . . . . . . . . . . . . 76 


\section{List of Tables}

2.1 Substrate Model parameters . . . . . . . . . . . . . . . 20

3.1 Phase relationship of lines $\ldots \ldots \ldots \ldots$

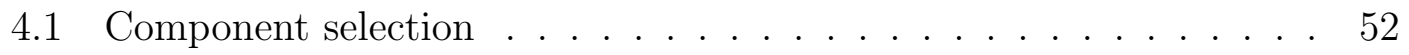

5.1 Resistor model parameters for a given nominal component value. Measurements done on VNA. . . . . . . . . . . . . . . . 62

5.2 Comparison of resistor values obtained by using the LCR meter and those obtained using VNA measurements. LCR measurements were done from $300 \mathrm{KHz}$ to $2 \mathrm{MHz}$ and the VNA data in the same frequency range was compared. Average of these sets of measurements is shown. . . . . . . . . . . . . . . . . . . . 64 64

5.3 Inductor model parameters for a given nominal component value. Measurement done on VNA. . . . . . . . . . . . . . 66

5.4 Comparison of inductor values obtained by using the LCR meter and those obtained using VNA measurements. LCR measurements were done from $300 \mathrm{KHz}$ to $2 \mathrm{MHz}$ and the VNA was measured at these same frequencies. Average of these sets of measurements is shown. . . . . . . . . . . . . . . . . . . 68

5.5 Capacitor model parameters for a given nominal component value. Measurement done on VNA. . . . . . . . . . . . . . . . . . . 69 
5.6 Comparison of capacitor values obtained by using LCR meter and those obtained using VNA measurements. LCR measurements were done from $300 \mathrm{KHz}$ to $2 \mathrm{MHz}$ and the VNA was measured at these same frequencies. Average of these sets of measurements is shown. . 71 


\section{List of Figures}

2.1 Two port network . . . . . . . . . . . . . . . . . 9

2.2 Vector representation in a complex reference plane . . . . . . . . . 11

2.3 Resonator Structure . . . . . . . . . . . . . . . . . . . . . . . 19

2.4 Resonator model vs actual measurement . . . . . . . . . . . 20

2.5 Southwest Connector layout . . . . . . . . . . . . . 22

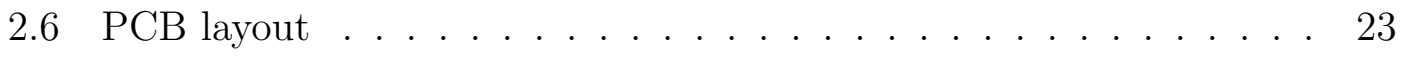

2.7 П Model . . . . . . . . . . . . . . . . . . . . . . . . . . 24

2.8 Resistor Model . . . . . . . . . . . . . . . . . . . . . . . 24

2.9 Simple Resistor Model . . . . . . . . . . . . . . . . . . 25

2.10 Capacitor Equivalent Model . . . . . . . . . . . . . . 25

2.11 Capacitor Practical Model . . . . . . . . . . . . . . 26

2.12 Large Inductor Model . . . . . . . . . . . . . . . . . 26

2.13 Small Inductor Model . . . . . . . . . . . . . . . . . . . 27

3.1 PCB Measurements . . . . . . . . . . . . . . . . . . 31

3.2 Calibration Lines . . . . . . . . . . . . . . . . . . 37

3.3 WinCal's Main Window . . . . . . . . . . . . . . . . . 39

3.4 WinCal's Setup Window . . . . . . . . . . . . . . 40

3.5 VNA Stimulus Settings . . . . . . . . . . . . . . . 41

3.6 Calibration Window $\ldots \ldots \ldots \ldots$. . . . . . . . 41

3.7 Calibration Setup Window . . . . . . . . . . . . . . . . . 42

3.8 TRL calibration settings $\ldots \ldots \ldots \ldots \ldots$

3.9 Measurement Window . . . . . . . . . . . . . . . . 43 
4.1 Measurement Setup 1 . . . . . . . . . . . . . . . . . 46

4.2 Measurement Setup 2 . . . . . . . . . . . . . . . . 48

4.3 ADS optimization example - $22 \mathrm{nH}$ Inductor . . . . . . . . . . 53

4.4 Comparison of extracted model (red), measured component (blue) and nominal model (black) values for S11 and S21 of $22 \Omega$ resistor. $\quad 55$

4.5 Smith chart illustration of frequency dependence of S11 and S22 for measured component (pink and black) and extracted model (red and blue) for $22 \Omega$ resistor. . . . . . . . . . . . 56

4.6 Comparison of extracted model (red), measured component (blue) and nominal model (black) values for S11 and S21 of $33 \mathrm{pF}$ capacitor. 57

4.7 Smith chart illustration of frequency dependence of S11 and S22 for measured component (pink and black) and extracted model (red and blue) for $33 \mathrm{pF}$ capacitor. . . . . . . . . . . . . . 58

4.8 Comparison of extracted model (red), measured component (blue) and nominal model (black) values for S11 and S21 of $22 \mathrm{nH}$ inductor. 59

4.9 Smith chart illustration of frequency dependence of S11 and S22 for measured component (pink and black) and extracted model (red and blue) for $22 \mathrm{nH}$ inductor. . . . . . . . . . . 60

5.1 Resistor percentage error with respect to nominal value for three samples. . . . . . . . . . . . . . . . . 62

5.2 Comparison of average value of resistors measured using LCR and

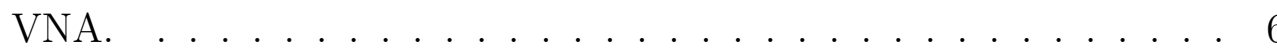

5.3 Inductor percentage error with respect to nominal value for three

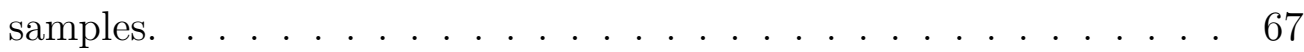


5.4 Comparison of average value of $22 \mu \mathrm{H}$ inductor measured using LCR

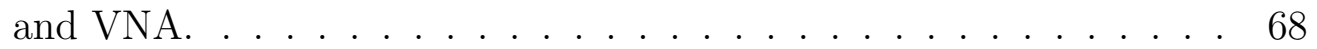

5.5 Capacitor percentage error with respect to nominal value for three

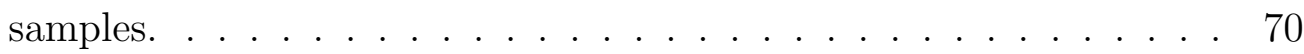

5.6 Comparison of average value of capacitors measured using LCR and

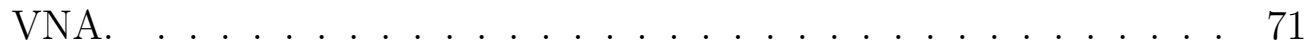




\section{Chapter 1}

\section{Introduction}

\section{$1.1 \quad$ Introduction}

This introduction gives a brief overview of the major parts of my thesis work: passive Surface Mount Devices (SMD), modeling, measurement and substrates. In today's high speed technology driven market, as the size of the electronic devices is shrinking, it is necessary to understand the behavior of passive SMD components. Nowadays, the use of traditional resistors, capacitors and inductors with leads is diminishing and the focus is more on SMD's. They have many advantages like small size and smaller parasitic power dissipation, as compared to traditional passive components. Also as the speed of transistors is getting faster and their size is getting smaller, the limitation now is not the logic but the interconnects. So it is very important to understand the behavior of these passive components in the transmission line environment.

In order to understand the behavior of SMD components in the real world, accurate models have to be developed so that simulation results will be comparable to the actual measured results. Modeling of these components becomes very difficult as the frequency increases due to the various parasitics, such as unwanted inductance in resistors, unwanted resistance in capacitors and unwanted capacitance in inductors, that come into effect at $\mathrm{GHz}$ frequencies.

At high frequencies the measurement itself becomes problematic and to ensure

the accuracy of measurements calibration is performed. Due to the high cost of the measuring instruments, cables, fixtures etc., we have to find ways to achieve 
calibration in a cost effective manner that will give us the desired accuracy. Over the years, calibration algorithms have been developed in order to take into consideration all the necessary parameters that might affect the measurements in transmission line environment.

As faster systems are developed, new substrates are constantly developed that support high frequency circuits and measurements. These new substrates are expensive and difficult to procure so other methods and substrates need to be developed if cost is the primary concern. One of the traditional substrates for manufacturing printed circuit board (PCB) is FR4 (flame retardant). It is a very common material used for manufacturing PCB's in the industry. This material is inexpensive and readily available in the market. This thesis focuses on how and when we can use inexpensive FR4 PCB for characterization of passive SMD's.

\subsection{Motivation}

In order to characterize the passive surface mount devices for research and academic purposes there is a need for a calibration kit which will be easy to design and manufacture. The other important factor to consider is the cost of this calibration kit. This thesis gives one of the possible solutions for developing the calibration kit using inexpensive FR4 substrates. This will be used for research and development as well as for academic laboratory purposes so that industry professionals and students can use this calibration kit for performing measurements of passive SMD components. The microstrip transmission lines which are used in the design are such that they have $50 \Omega$ characteristic impedance. This is determined by calculating the width of the microstrip lines to get $50 \Omega$ using, the LineCalc tool in Agilent's Advanced Design System (ADS) microwave simulation software. Recent 
industry practice is to use the impedance controlled lines so that they can have constant impedance on the transmission line. We are exploring an inexpensive way of doing the same.

Bench test equipment can be used to build and characterize this calibration kit. This involves using many lab testing instruments like the Time Domain Reflectometery (TDR), Vector Network Analyzer (VNA), LCR meter, Agilent's Electronic Calibration kit (ECAL), high precision digital multimeter and Vernier calipers.

The PCB which was used in this thesis was manufactured by Advanced Circuits (www.4pcb.com). Also the resistor and capacitor models were developed by our collaborators at the University of Zagreb while we developed the inductor model. These are simple models which are used to simulate real world behavior of these

passive SMD components. Extensive optimization in ADS was done so as to fit this modeled data to the measured data.

\subsection{Need for calibration}

The RF test system consists of the test system interface to the Device Under Test (DUT) (either coplanar probes or a test fixture), VNA and the connecting cables. Errors like drift error, random error and systematic error cause the measurement results to be inaccurate. Calibration serves to quantify the degree of error in the test system. The errors found in the RF test system can be mathematically removed by applying error models. Different models of RF calibration are available for example, SOLT, TRL, LRM etc., each based on measuring a set of standards in place of the DUT. By measuring the standards mounted in place of the DUT, the test systems imperfections can be isolated, quantified and mathematically removed. 
Calibration de-embeds imperfections in the test system by measuring known quantities called standards. Different calibration algorithms are developed depending on the standards used. For this thesis TRL calibration was used to get accurate and repeatable measurements.

\subsection{Accurate modeling of SMD devices - Necessity}

As we start going higher in frequencies the passive components do not behave as they are supposed to due to the parasitics that come into picture. Some times it is necessary to push the components even outside of the frequency range that they were designed for. In such cases, details of device behavior, including the parasitics, become very important and we need to model the device accurately. In other cases, such as biasing networks, device ac behavior can be neglected at lower frequencies but we have to account for their changing impedance at high $(\mathrm{GHz})$ frequencies. Furthermore, the actual environment (substrate) in which devices operate can influence their modeling and accurate models are necessary for any hope of first-pass success [9].

\subsection{Thesis contribution}

This work focuses on developing fixtures and TRL calibration kit based on microstrip lines, made from inexpensive FR4 material used for characterizing the surface mount devices. The passive SMD components were measured using the LCR meter. Results of the LCR meter were compared with the results obtained from the VNA measurements. Simple models that were developed for the passive SMD components like the resistors, capacitors and inductors were sufficiently accurate up to $4 \mathrm{GHz}$. 
This work examines the range of operating frequency of the SMD components. A range of values of the resistors, capacitors and inductors were measured and modeled. Optimization was performed in ADS to fit the modeled data to the measured data.

\subsection{Thesis layout}

This thesis is laid out in six chapters.

Chapter 2 discusses the essential microwave theory as well as the simple resistor, capacitor and inductor models. This chapter also explains the resonator structure which is used for the validation of the FR4 substrate.

Chapter 3 discusses the standards and calibration algorithm. The WinCal XE software which is used for performing the calibration is discussed as well.

Chapter 4 describes the measurement setup and optimization algorithm used in ADS.

Chapter 5 shows a comparison of the measurement results with the modeled results.

Chapter 6 discusses the conclusions and the scope for future work. 


\section{Chapter 2}

\section{Background and Theory}

\subsection{Scattering Parameters [S-parameters]}

In order to characterize linear devices the parameters like H-, Y- and Z-parameters are used but as the frequencies at which the devices operate become higher it becomes difficult to use these parameters for characterization. These parameters need open and short circuits for making the measurements so that the currents and voltages in the circuit can be calculated. For example, consider a two port network, in order to calculate the input impedance of the network we have to apply a short circuit at the output port of the network. There is a problem with these measurements at higher frequencies. Equipment is not readily available to measure total voltage and total current at the ports at the network. Also, short and open circuits are difficult to achieve over a broad band of frequencies. Many active devices, such as transistors and tunnel diodes, very often will not be short or open circuit stable. The logical variables to use at these frequencies are traveling waves rather than total voltages and currents [1].

\subsection{Transmission Lines}

All high frequency systems have a source of power. A portion of this power is delivered to a load by means of transmission lines.

Consider a transmission line environment in which $Z_{s}$ is the source impedance and $Z_{0}$ is the characteristic impedance of the transmission line. Voltage, current and power can be considered to be in the form of waves traveling in both directions 
along this transmission line. A portion of the waves incident on the load will be reflected. It then becomes incident on the source, and in turn it re-reflects from the source, if $Z_{s} \neq Z_{0}$, resulting in a standing wave on the line [1].

If this transmission line is uniform in cross section, it can be thought of as having an equivalent series impedance and equivalent shunt admittance per unit length. A lossless line would simply have a series inductance and a shunt capacitance. The characteristic impedance of the lossless line, $Z_{0}$, is defined as shown in eq. 2.1 .

$$
Z_{0}=\sqrt{\frac{L}{C}}
$$

One useful parameter is the voltage reflection coefficient $\Gamma$, as shown in eq. 2.2. It relates the load impedance $\left(Z_{L}\right)$ to the characteristic impedance $\left(Z_{0}\right)$ of the transmission line. This is the measure of the quality of the impedance match between the load and the characteristic impedance of the line. The reflection coefficient is a complex quantity having a magnitude, $\rho$ and angle, $\theta$. The better the match between the load and the characteristic impedance of the line, the smaller the reflected voltage wave and the smaller the reflection coefficient.

$$
\Gamma=\frac{Z_{L}-Z_{0}}{Z_{L}+Z_{0}}
$$

\subsection{Definition of S-parameters}

We are used to dealing with total voltages and total currents but since it is difficult to measure these quantities at higher frequencies we define a new set of parameters/functions for a two port network relating the incident and reflected traveling waves at the network ports i.e. the traveling voltage waves.

This new set of parameters or functions are called "Scattering parameters" 
since they relate those waves scattered or reflected from the network to those waves incident upon the network. These are also known as S-parameters.

S-parameters are a means for characterizing linear n-port networks. S-parameters do not use open or short circuit conditions to characterize a linear electrical network; instead matched loads are used. These matched load terminations are much easier to use at high signal frequencies than open-circuit and short-circuit terminations. Moreover, the quantities are measured in terms of power.

S-parameters change with:

- Measurement frequency

- Characteristic impedance or

- System impedance

For a 2-port network the S-parameters used are S11, S12, S21 and S22. These represent reflection and transmission coefficients of the two port network, measured at port 1 and 2, as shown in Figure 2.1.

$$
[\mathrm{S}]=\left[\begin{array}{ll}
S 11 & S 12 \\
S 21 & S 22
\end{array}\right]
$$

The S-parameters relate the input and output through the S-parameter matrix. The equations are described as below.

$$
B 1=S 11 A 1+S 12 A 2
$$




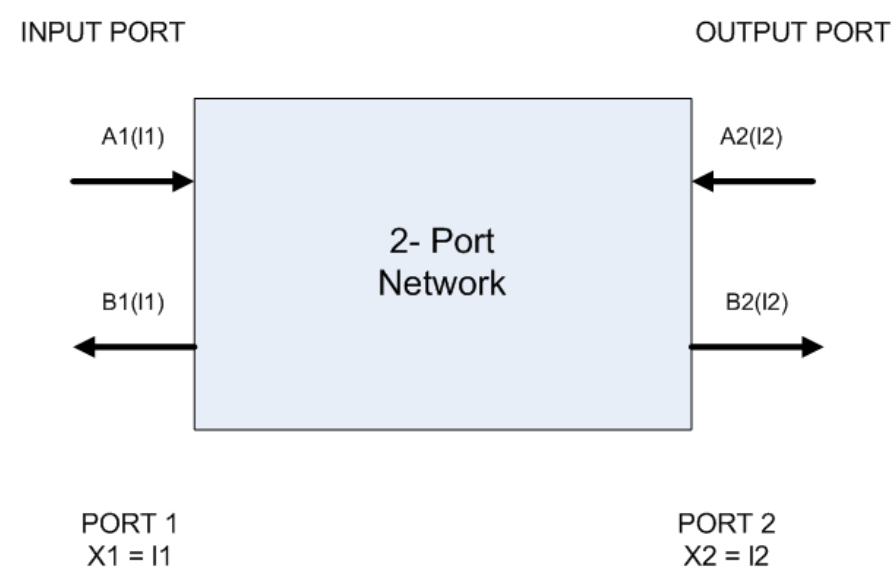

Figure 2.1: Two port network

$$
B 2=S 21 A 1+S 22 A 2
$$

\subsubsection{S-parameter equations}

The S parameters are seen to represent reflection and transmission coefficients. The S parameters measured at the specific locations shown at port 1 and port 2 are defined as follows:

Input reflection coefficient with output port terminated with a matched load is given by eq. 2.5 .

$$
S 11=\left.\frac{B_{1}\left(l_{1}\right)}{A_{1}\left(l_{1}\right)}\right|_{A_{2}\left(l_{2}\right)_{=0}}
$$

Forward transmission coefficient with output matched is given by eq. 2.6.

$$
S 21=\left.\frac{B_{2}\left(l_{2}\right)}{A_{1}\left(l_{1}\right)}\right|_{A_{2}\left(l_{2}\right)=0}
$$

Output reflection coefficient with input port terminated with a matched load is given by eq. 2.7 . 


$$
S 22=\left.\frac{B_{2}\left(l_{2}\right)}{A_{2}\left(l_{2}\right)}\right|_{A_{1}\left(l_{1}\right)_{=0}}
$$

Reverse transmission coefficient with input matched is given by eq. 2.8.

$$
S 12=\left.\frac{B_{1}\left(l_{1}\right)}{A_{2}\left(l_{2}\right)}\right|_{A_{1}\left(l_{1}\right)_{=0}}
$$

Therefore, from eq. 2.5, the term $\mathrm{S} 11 \cdot \mathrm{A} 1(11)$ represents the contribution to the reflected wave B1(11) due to incident wave A1(11) at port 1. Similarly, from eq. 2.8, S12·A2(12) represents contribution to reflected wave B1(11) due to incident wave A2(12) at port 2 and so on.

The S-parameters tell us how much power is absorbed, reflected or transmitted by the DUT. This gives us a complete description of the DUT. For example, on a transmission line with $Z_{0}=50 \Omega$ if the device is a $50 \Omega$ resistor then all the power waves that are sent from the source will be absorbed by this $50 \Omega$ resistor and there will be no reflections. On the other hand if this resistor is not $50 \Omega$ then there will be reflections which will help us to ultimately characterize the devices under test; in the present case these are the resistors, capacitors and inductors.

\subsection{Impedance Measurement Basics}

Impedance

1. Impedance is an important parameter used to characterize electronic circuits, components and the materials used to make components. Impedance $(\mathrm{Z})$ is generally defined as the total opposition a device or circuit offers to the flow of an Alternating Current (AC) at a given frequency, and is represented as a complex quantity which is graphically shown on a complex plane [4]. 


\section{Imaginary axis}

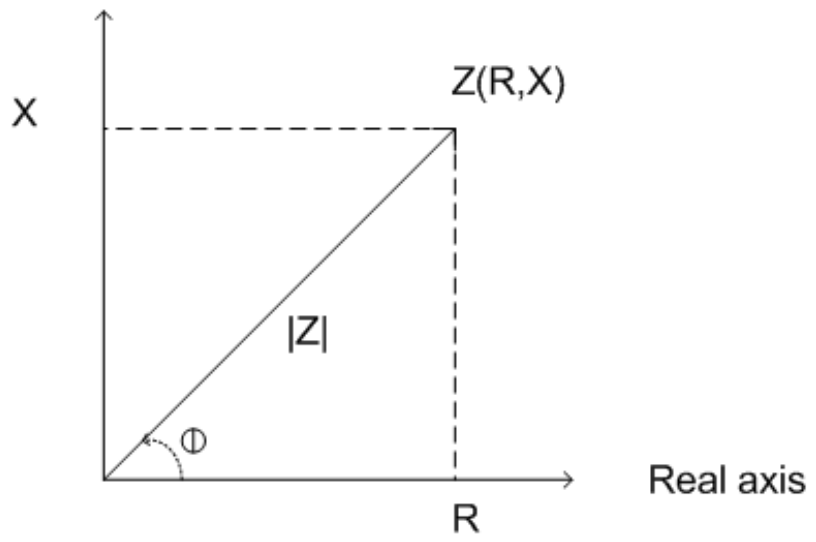

Figure 2.2: Vector representation in a complex reference plane

2. An impedance vector consists of a real part (resistance $R$ ) and an imaginary part (reactance $\mathrm{X}$ ) as shown in Figure 2.2.

3. Impedance can be expressed using the rectangular coordinate form

$$
R+j X
$$

or in the polar form as a magnitude and phase angle

$$
|Z| \angle \theta
$$

4. Reactance takes two forms

- Inductive $X_{L}$

$$
X_{L}=2 \pi f L
$$


and

- Capacitive $X_{C}$

$$
X_{C}=\frac{1}{(2 \pi f C)}
$$

5. The quality factor $(\mathrm{Q})$ serves as a measure of a reactance's purity (how close it is to being a pure reactance, no resistance), and is defined as the ratio of the energy stored in a component to the energy dissipated by the component. $\mathrm{Q}$ is a dimensionless unit and is expressed as

$$
Q=\frac{X}{R}=\frac{B}{G}
$$

where,

$$
\begin{aligned}
& \mathrm{X} \rightarrow \text { Reactance, } \\
& \mathrm{R} \rightarrow \text { Resistance, } \\
& \mathrm{B} \rightarrow \text { Susceptance, } \\
& \mathrm{Y} \rightarrow \text { Admittance }
\end{aligned}
$$

\subsubsection{Ideal, real and measured values}

When we determine an impedance parameter value for a circuit component (resistor, capacitor or inductor), it is important to thoroughly understand what the value indicates in reality.

\section{Ideal}

An ideal value is the value of a circuit component (resistor, inductor or capacitor) that excludes the effects of its parasitics. The model of an ideal component assumes 
a purely resistive or reactive element that has no frequency dependence. In many cases, the ideal value can be defined by a mathematical relationship involving the component's physical composition [11], such as

Resistance

$$
R=\rho \frac{l}{A}
$$

where,

$\mathrm{R} \rightarrow$ Resistance

$\rho \rightarrow \operatorname{Resistivity~}(\Omega \mathrm{m})$

$l \rightarrow$ length of the specimen in meters $(\mathrm{m})$

$\mathrm{A} \rightarrow$ cross sectional area of the specimen in square meters $\left(\mathrm{m}^{2}\right)$

Capacitance (Parallel plate capacitor)

$$
C=\epsilon_{r} \epsilon_{0} \frac{A}{d}
$$

where,

$\epsilon_{r} \rightarrow$ relative static permittivity, also known as dielectric constant (for vacuum, $\epsilon_{r}$ $=1$ )

$\epsilon_{0} \rightarrow$ dielectric constant $\left(\epsilon_{0}=8.854 \times 10^{-12} \mathrm{~F} \cdot \mathrm{m}^{-1}\right)$

$\mathrm{d}=$ separation between the plates

$A=$ area of overlap of the two plates 
Inductor (inductance of a cylindrical air core coil)

$$
L=\frac{\mu_{0} K N^{2} A}{l}
$$

where,

$\mathrm{L} \rightarrow$ inductance in henries $(\mathrm{H})$

$\mu_{0} \rightarrow$ permeability of free space $=4 \times 10^{-7} \frac{\mathrm{H}}{\mathrm{m}}$

$\mathrm{K} \rightarrow$ Nagaoka coefficient[6]

$\mathrm{N} \rightarrow$ number of turns

$\mathrm{A} \rightarrow$ area of cross-section of the coil in square meters $\left(\mathrm{m}^{2}\right)$

$l \rightarrow$ length of coil in meters $(\mathrm{m})$

\section{Real}

The real value of a circuit component takes into consideration the effects of a component's parasitics. The real value represents effective impedance, which a real-world component exhibits. The real value is the vector sum of the circuit component's resistive and reactive vectors, which come from the principal element (deemed as pure element) and the parasitics. Since the parasitics yield a different impedance vector for a different frequency, the real value is frequency dependent [4].

\section{Measured}

The measured value is the value obtained with, and displayed by, the measurement instrument; it reflects the instrument's inherent residuals and inaccuracies. Measured values always contain errors when compared to real values. They also vary intrinsically from one measurement to another; their differences depend on a multitude of considerations in regard to measurement uncertainties. We can judge 
the quality of measurements by comparing how closely a measured value agrees with the real value under a defined set of measurement conditions. The real value is what we want to know, and the goal of the measurement is to have the measured value be as close as possible to the real value.

\subsection{Parameters that affect the measurement system}

The essential elements in an RF test system are:

1. The test system interface to the Device Under Test(DUT) (either coplanar probes or a test fixture)

\section{VNA}

\section{Connecting Cables}

In order to measure SMD components accurately, it is necessary to understand the factors contributing to the measurement errors in the measurement system. Nonidealities exist in the test system and to correct for these, test fixtures must

be calibrated. In the following section we will utilize [6], [8] and [3] to get more information about the measurement system.

\subsubsection{Test System Errors}

\section{Drift Errors}

Drift errors are caused by deviations in the performance of the measuring instrument that occur after calibration. Major causes are the thermal expansion of connecting cables and thermal drift of the frequency converter within the measuring instrument. These errors may be reduced by carrying out 
frequent calibrations as the ambient temperature changes or by maintaining a stable ambient temperature during the course of measurement.

\section{Random Errors}

Random errors occur irregularly in the course of using the instrument. Since random errors are unpredictable, they cannot be completely eliminated by calibration. These errors are further classified into the following subcategories depending on their causes.

- Instrument noise errors

These errors are caused by electrical fluctuations within components used in the measuring instrument in VNA's. These errors may be reduced by increasing the power of the signal applied to the DUT, narrowing the Intermediate Frequency (IF) bandwidth, or enabling sweep averaging.

- Switch repeatability errors

These errors occur due to the fact that the electrical characteristics of the mechanical Radio Frequency (RF) switch used in the measuring instrument change every time it is switched on. These errors may be reduced by carrying out measurements under conditions in which no switching operation takes place.

- Connector repeatability errors

These errors are caused by fluctuations in the electrical characteristics of connectors due to wear. These errors may be reduced by handling connectors with care. 


\section{Systematic Errors}

Systematic errors are caused by imperfections in the measuring instrument and the test setup (cables, connectors, fixtures, etc). Assuming that these errors are repeatable (i.e. predictable) and their characteristics do not change with time, it is possible to eliminate them mathematically at the time of measurement by determining the characteristics of these errors through calibration. There are six types of systematic errors, they are as follows [6].

- Errors caused by signal leaks in the measuring system

- Directivity: Directivity errors are caused by the fact that, in a reflection measurement, signals other than the reflection signal from the DUT are received by receiver through the directivity coupler.

- Isolation (cross-talk): Isolation errors are caused by signals other than the transmission signal of the DUT leaking to the test receiver of the transmission measurement port.

- Errors caused by reflections in the measuring system

- Source match: A source match error is caused when the reflection signal of the DUT reflects at the signal source and enters the DUT again.

- Load match: A load match error is caused when part of the signal transmitted in the DUT reflects at a response port and is measured by the receiver of the response port.

- Errors caused by the frequency response of the receiver within the measuring instrument 
- A reflection tracking error is caused by the difference in frequency response between the test receiver and the reference receiver of a stimulus port.

- A transmission tracking error is caused by the difference in frequency response between the test receiver of a response port and the reference receiver of a stimulus port.

\subsection{PCB}

The Printed Circuit Board (PCB) is a major limiting factor when we take high frequency measurements. Important factors that affect the system performance are the dielectric constant of the FR4 substrate and the thickness of the PCB. The

resonator structure was used to ensure the validity of the dielectric constant of the substrate provided by the manufacturer. Also the thickness of the conducting microstrip transmission line and the substrate affect the performance of the measurements drastically. We used the Vernier calipers to measure the thickness of the substrate as well as the thickness of the tin plated copper microstrip transmission lines.

\subsection{Resonator structure}

The resonator structure is used to validate the dielectric constant of the FR4 substrate of the PCB and is shown in the Figure 2.3. The design of the resonator structure is based on [7].

The fixture in the initial design consisted of an Edge mount SMA jack of $50 \Omega$ from Johnson components. The first version of the PCB was used to validate the dielectric of the FR4 substrate provided by the PCB manufacturer. The PCB's 


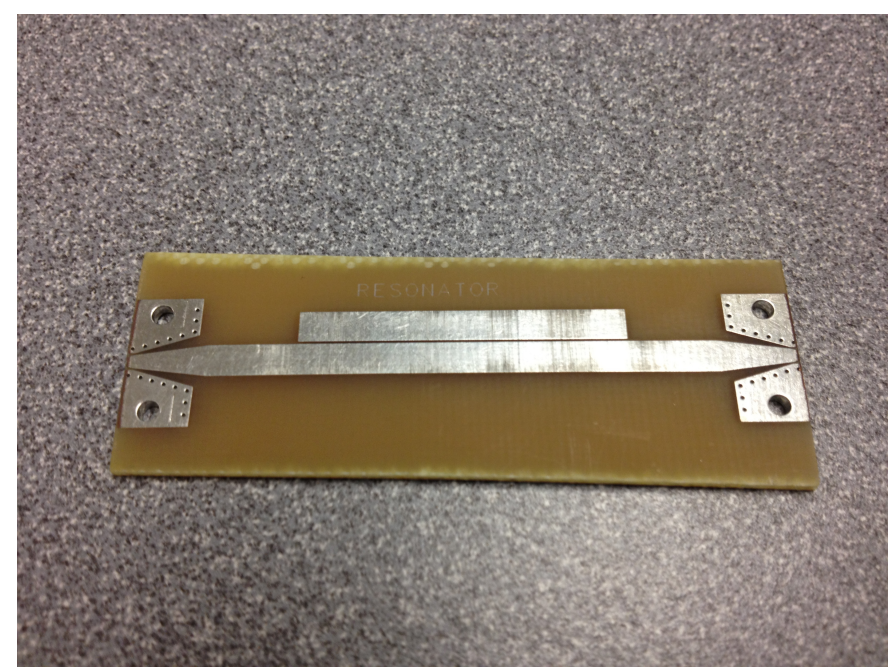

Figure 2.3: Resonator Structure

were made by Advanced Circuits (www.4pcb.com) located in Arizona.

The resonator circuit is called the microstrip line parallel-coupled resonator and is made of two coupled microstrip lines as shown in Figure 2.3. The circuit was initially simulated in ADS simulator. Then Momentum simulation for the circuit was performed to see the behavior of the electromagnetic coupling between these two lines. Momentum is an EM simulator based on the method of moments. A substrate model was defined in ADS as per the substrate definition provided by Advanced Circuits. This model consisted of substrate thickness, dielectric constant, conductivity of the copper material, thickness of the microstrip line and loss tangent. The resonator circuit was simulated in ADS and compared with the measured VNA data. This resonator circuit model was used to extract the dielectric constant at the frequencies of zeros in the measured transmission response as shown in Figure 2.4. In this Figure 2.4 the modeled data is shown in red and the measured data is shown in black. By adjusting the model's dielectric constant, we tuned the frequency of each zero to match the measured frequency, yielding the dielectric constant at that frequency. Finally, after tuning the parameters of the 

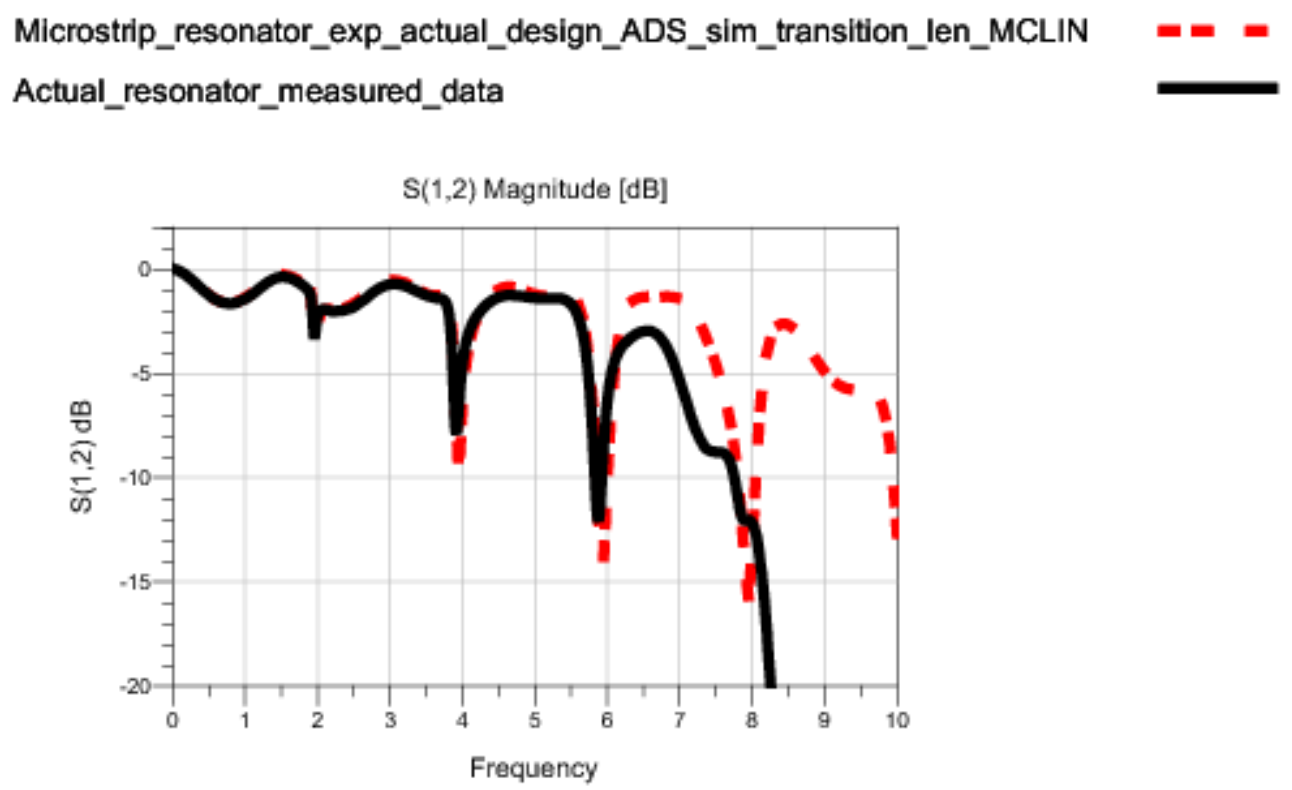

Figure 2.4: Resonator model vs actual measurement

substrate model, the parameters that were used are shown in Table 2.1.

There were problems using the edge mount connector since we have to solder the connector at five different places which makes it hard to reuse. To overcome this problem we used the panel mount, square flanged SMA STR connector by Hirose Electric Company Limited (Digikey Part No - H11565-ND). This connector had to be screwed to an aluminum base plate so that the body of the connector

\begin{tabular}{|l|c|}
\hline Parameter & Value \\
\hline Substrate thickness $(\mathrm{H})$ & $1.56 \mathrm{~mm}$ \\
\hline Dielectric constant $\left(\epsilon_{r}\right)$ & 4.25 \\
\hline Mur & 1 \\
\hline Conductivity of copper & $6 \mathrm{e}+7$ \\
\hline Thickness of copper & $0.04064 \mathrm{~mm}$ \\
\hline TanD & 0.016 \\
\hline Cover height & $3.9 \mathrm{e}+034 \mathrm{mil}$ \\
\hline
\end{tabular}

Table 2.1: Substrate Model parameters 
could be grounded to the ground of the PCB. Due to the dimensions of the base plate that was used for this connector it was difficult to mount this connector on the PCB and take measurements. To overcome this problem we had to search for a better and more reliable connector and we finally settled on the Southwest Microwave Connector. This is an end launch connector which can be screwed to the PCB. It is a useful feature which is very helpful for reusing the connector.

In the third and final revision of the PCB we used high precision end launch connectors provided by Southwest Microwave. The layout of the transition is shown in Figure 2.5. These connectors were easy to work with and they work well up to tens of GHz. Also the layout of the complete PCB which was manufactured by Advanced Circuits is shown in Figure 2.6.

\subsection{Reference $\Pi$ Model}

Figure 2.7 shows the reference $\Pi$ model that is used for the representation of modeling the passive SMD circuits. It consists of two main parts: pad capacitances $C_{p a d}$ on each end and the core central model that depends on the type of the device modeled. In order to estimate the effect of SMD soldering pads we can calculate the parallel plate capacitance using eq. 2.17.

Capacitance (Parallel plate capacitor)

$$
C=\epsilon_{r} \epsilon_{0} \frac{A}{d}
$$

where,

$\epsilon_{r} \rightarrow$ relative static permittivity, also known as dielectric constant (for FR4, $\epsilon_{r}=$ $4.25)$ 


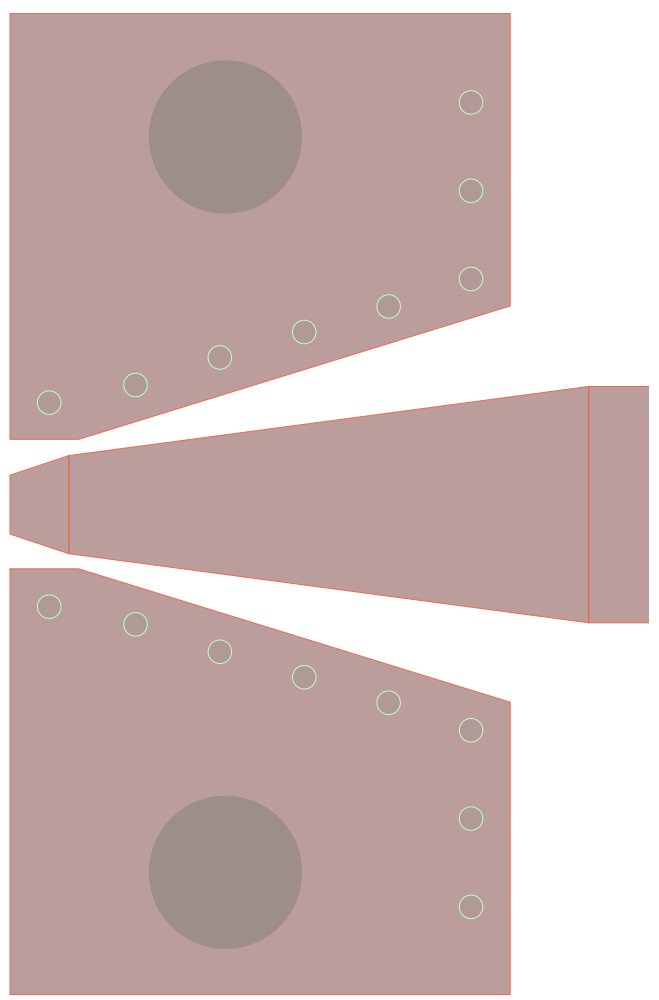

Figure 2.5: Southwest Connector layout 


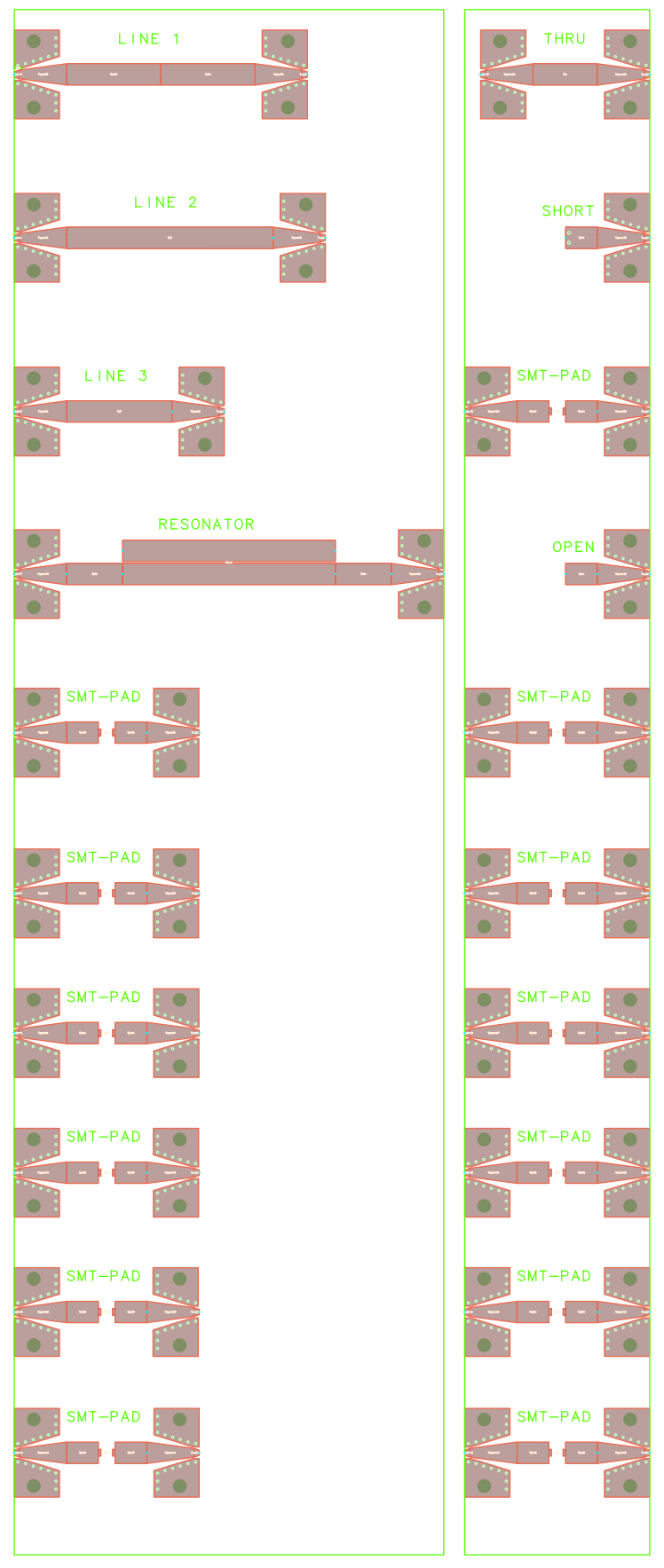

Figure 2.6: PCB layout 


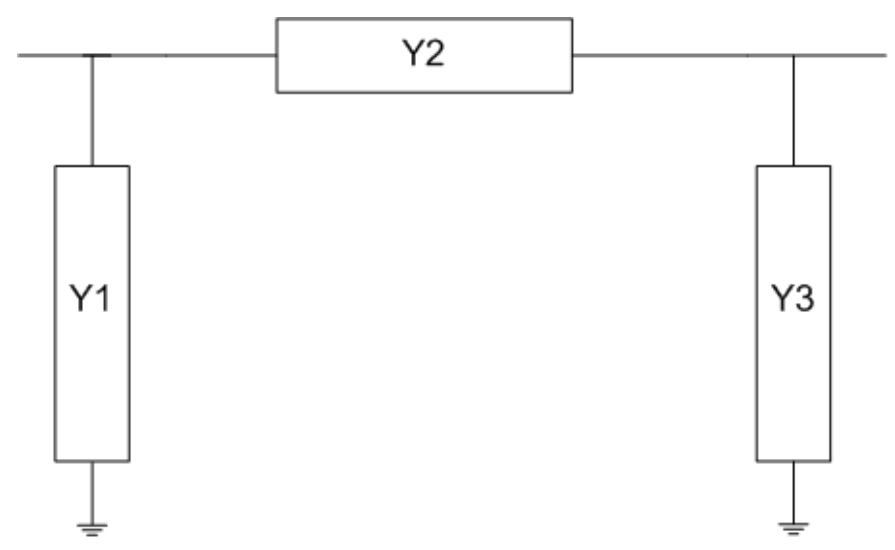

Figure 2.7: ПI Model

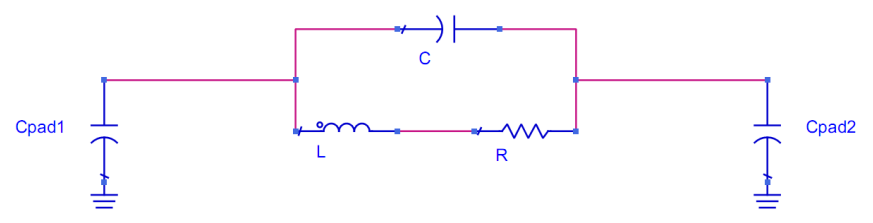

Figure 2.8: Resistor Model

$\epsilon_{0} \rightarrow$ electric constant $\left(\epsilon_{0}=8.854 \times 10^{-12} \mathrm{~F} \cdot m^{-1}\right)$

$\mathrm{d}=$ separation between the plates (substrate thickness $=1.56 \mathrm{~mm}$ )

$\mathrm{A}=$ area of overlap of the two plates $($ pad dimensions $=(0.98 \mathrm{~mm} \times 0.35 \mathrm{~mm}))$ After applying this parallel plate capacitance formula, we get the approximate pad capacitances to be equal to $8.3 \times 10^{-15} \mathrm{~F}$. Note that the SMD package itself may have additional parasitic "pad" capacitance.

\subsection{Resistor - Simple Models}

The equivalent circuit model of a resistor is shown in Figure 2.8.

For frequencies that are well below resonant frequency, $\mathrm{L}$ and series $\mathrm{R}$ are dominant circuit elements. Since we are operating in $\mathrm{GHz}$ frequency range we can use the model, as shown in Figure 2.9. In this model a shunt capacitance $\left(C_{r}\right)$ and 


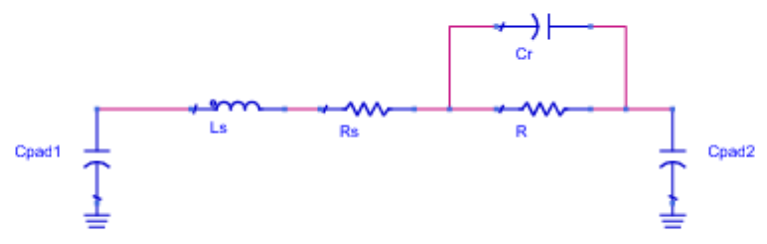

Figure 2.9: Simple Resistor Model

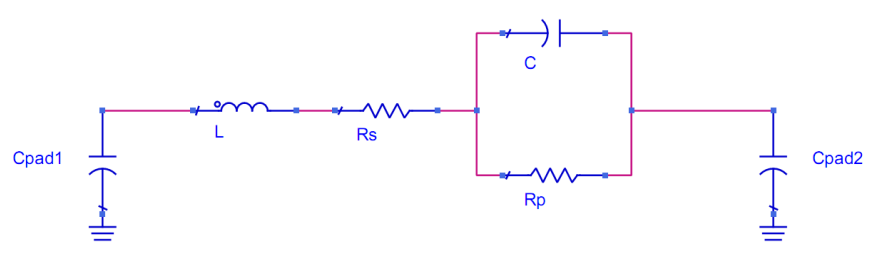

Figure 2.10: Capacitor Equivalent Model

series inductance $\left(L_{s}\right)$ is used since we are operating at $\mathrm{GHz}$ frequency range.

\subsection{Capacitor - Simple Models}

Capacitance (C), dissipation factor (D) and Equivalent Series Resistance (ESR) are the parameters generally measured.

A practical capacitor equivalent circuit is shown in Figure 2.10. C denotes the main element of the capacitor. Rs and $\mathrm{L}$ are the residual resistance and inductance existing in the lead wires and electrodes. $\mathrm{Rp}$ is a parasitic resistance which represents the dielectric loss of the dielectric material. The $\mathrm{Rp}$ is usually insignificant and can be disregarded in cases of high-value capacitors (because $\operatorname{Rp} \gg \frac{1}{2 \pi f C}$ ). For low-value capacitors, the Rp itself has an extremely high value. This gives us a more practical capacitor model as shown in Figure 2.11.

At low frequencies the phase angle $(\theta)$ of impedance is around -90 degrees, 


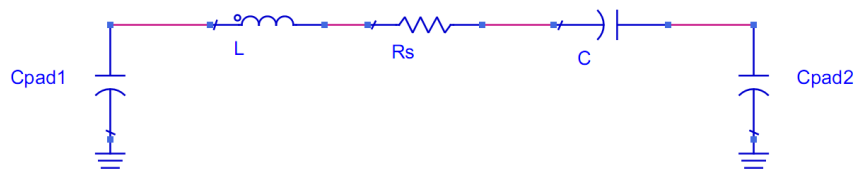

Figure 2.11: Capacitor Practical Model

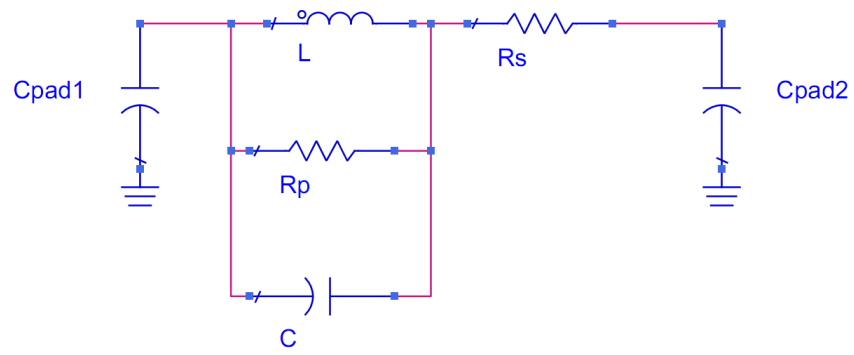

Figure 2.12: Large Inductor Model

so the reactance is capacitive. The capacitor frequency response has a minimum impedance point at a Self-Resonant Frequency (SRF), which is determined from the capacitance and parasitic inductance (Ls) of a series equivalent circuit model of the capacitor. At the self-resonant frequency, the capacitive and inductive reactance values are equal $\left(\frac{1}{2 \pi f C}=2 \pi f L s\right)$. As a result, the phase angle is 0 degrees and the device is resistive. After the resonant frequency, the phase angle changes to a positive value around +90 degrees and, thus, the inductive reactance due to the parasitic inductance is dominant. Capacitors behave as inductive devices at frequencies above the SRF and, as a result, cannot be used as a capacitor above the SRF.

\subsection{Inductor - Simple Models}

A typical equivalent circuit for an inductor is shown in Figure 2.12. In this figure, Rp represents the magnetic loss (which is called iron loss) of the inductor core, 


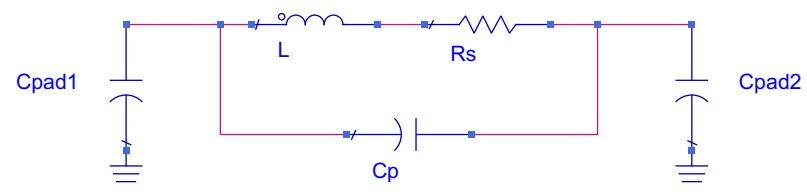

Figure 2.13: Small Inductor Model

and Rs represents the copper loss (resistance) of the wire. C is the distributed capacitance between the turns of the wire. For small inductors the equivalent circuit shown in Figure 2.13 can be used. This is because the value of $\mathrm{L}$ is small and the stray capacitance between the lead wires (or between the electrodes) becomes a significant factor. This model fits many SMD (chip) type RF inductors.

Since the reactance $(2 \pi f L)$ decreases at lower frequencies, the minimum impedance is determined by the resistance (Rs) of winding. The stray capacitance $\mathrm{Cp}$ is the prime cause of the inductor frequency response at high frequencies. The existence of $\mathrm{Cp}$ can be recognized from the resonance point, SRF, in the higher frequency region. At the SRF, the inductor exhibits maximum impedance because of parallel resonance $\left(2 \pi f L=\frac{1}{2 \pi f C p}\right)$ due to $\mathrm{Cp}$. After the resonance frequency, the phase angle of impedance is a negative value around -90 degrees because the capacitive reactance of $\mathrm{Cp}$ dominates. The measured inductance $(\mathrm{Lm})$ rapidly increases as the freq approaches the SRF because of the effect of resonance. The maximum Lm value becomes greater as the device has a higher $\mathrm{Q}$ factor. At frequencies above the 
SRF, a negative inductance value is displayed because the Lm value is calculated from a capacitive reactance vector, which is opposite to inductive vector.

Typically, the SRF determines the maximum usable frequency of capacitors and inductors. 


\section{Chapter 3}

\section{Calibration Methodology}

\subsection{Introduction to Calibration}

To ensure the validity of the measured data, the test equipment must be calibrated. For example, consider a VNA: it has connecting cables and test fixtures to go through before it can actually measure the Device Under Test (DUT); in our case it is the SMD component. To account for these errors we have to calibrate the instrument so as to achieve full confidence in the measured data. The calibration process employs a technique called vector error correction, in which error terms are characterized using known standards so that errors can be removed from actual measurements. The process of removing these errors requires the errors and measured quantities to be measured vectorially (thus the need for a vector network analyzer) $[10]$.

Calibration de-embeds imperfections in the test system by measuring known quantities called standards. By measuring the standards mounted in place of the DUT, the test system's imperfections can be isolated, quantified and mathematically removed.

\subsection{Reference Plane}

The line where the test system ends and the DUT begins is known as the reference plane. There are two different types of reference planes.

1. Physical Reference Plane 
Physical reference plane is a line which divides the test system and DUT physically. For example on a schematic if we are able to draw a line which divides the DUT from the test setup, this line is called the physical reference plane.

\section{Electrical Reference Plane}

When RF coupling occurs defining the electrical reference plane becomes difficult. For example, consider the male pin of a SMA connector, it is inside a housing, we can not draw a physical line at the edge of the male connector tip inside the housing. Similarly, for a female SMA connector we cant draw a line at the edge of the connector. The line where we can divide the system at the exact location where the connector starts is known as the electrical reference plane. After the male connector is mated into the female connector the electrical reference plane of this male connector goes beyond this female connector and so we have to perform calibration which will help us to move this electrical reference plane to the correct location.

\subsection{Calibration Standards}

A valid calibration involves measuring a series of physical elements called standards. A set of standards developed in this work are shown in Figure 3.1. Each standard is measured so as to get magnitude and phase of its impedance over frequency.

By measuring standards in place of DUT the RF path can be calibrated, deembedding the errors in the test setup. Most calibration techniques use a combination of standards such as a short circuit, an open circuit, a load and a through 


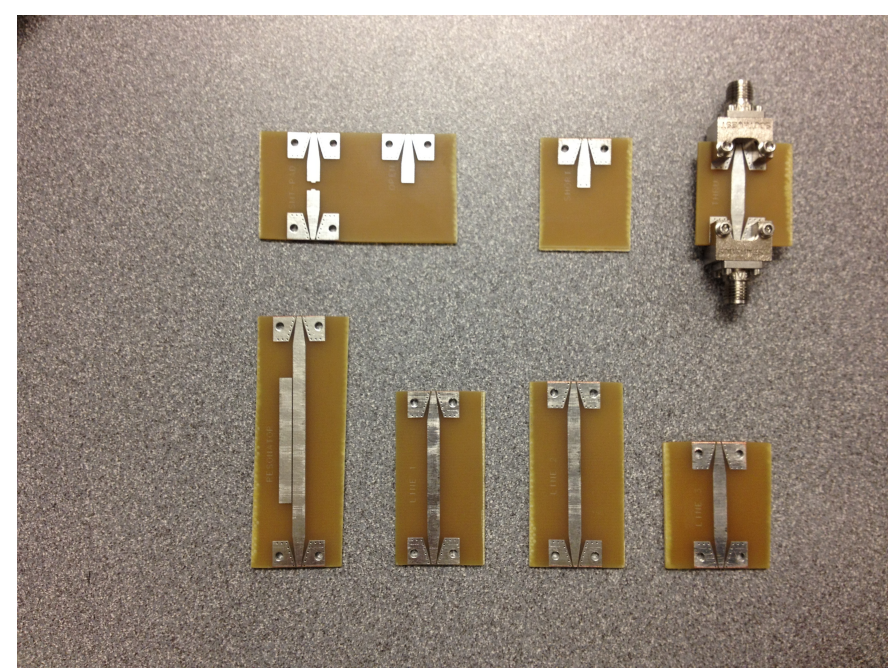

Figure 3.1: PCB Measurements

line. Combined, these remove the test setup's error.

\subsubsection{Short}

The ideal short totally reflects the incident signal so that its reflection coefficient $\Gamma=-1$. After impinging on the short, the incident signal changes phase 180 degrees and travels back towards the source. In practice, this short length acts like a parasitic inductor.

\subsubsection{Open}

The open makes no connection between the signal and ground, ideally reflecting the incident signal with no loss. An ideal open would yield a reflection coefficient $\Gamma$ of unity with 0 degrees of phase shift. In reality, electric fields connect the signal probe to the ground plane, forming parasitic capacitance. 


\subsubsection{Load}

The load is a terminating resistor mounted between signal and ground probes. When swept over frequency, the load trace ideally swirls about the center of the Smith chart. In reality, parasitic inductance arises due to the interconnecting pads at either end of the resistor. Loads greater than $100 \Omega$ tend to have larger amounts of shunt capacitance.

\subsubsection{THRU}

THRU connects the two RF test ports together. The THRU is typically a $50 \Omega$ transmission line that connects the two ports together. It is different from the short in a way that it connects the two ports together, while the short connects one port to the ground plane. There is quite an amount of phase change and attenuation once a signal passes from one port to another and this can be used for characterizing the calibration kit. For a non-zero length THRU, the center of the THRU determines the center of the reference plane.

\subsection{Basic calibration process}

The calibration process goes through the following steps:

Step 1: Design of the measuring instrument that needs to be calibrated. The design has to be capable of measurements that are "within engineering tolerance" when used within stated environmental conditions over some reasonable period of time. The measuring equipment manufacturer generally assigns the measurement

tolerance, suggests a calibration interval and specifies the environmental range of use and storage. 
Step 2: Defining the calibration process. The selection of a standard or standards is the most visible part of the calibration process. There may be specific connection techniques between the standard and the device being calibrated that may influence the calibration process. For example, in electronic calibration involving analog phenomena the impedance of the cable connection can directly influence the result. All of the information is collected in a calibration procedure, which constitutes a specific test method.

Factors that help to determine the PCB calibration

1. Material used for making the PCB

2. Structures which are used. For example:

- Microstrip line traces

- Stripline Transmission line

- Coplanar waveguide

\subsection{TRL (Thru-Reflect-Line) Calibration}

Transmission lines are simple to understand and easy to fabricate. Their physical dimensions and the board material decide their characteristic impedance. Because it is based on the transmission line standard, TRL calibration is a powerful method.

\subsubsection{THRU}

A short transmission line serves as the THRU. In general the line lengths leading to the DUT should be the same length as the THRU. The offset delay shifts the phase of the signal. When the offset delay is set to zero, the THRU's midpoint sets 
the electrical reference plane. Applying the offset delay is a simple way to shift the electrical phase to compensate for the pads.

\subsubsection{Reflect}

The reflect standard can be either an open or a short. Only the sign of its reflection coefficient needs to be known. The reflect does not have to be a perfect open or short, although the best results have a $\Gamma$ close to 1 . The reflect's phase should be generally known to within \pm 90 degrees. When selecting a reflect, one should keep in mind that an open operates over a broader bandwidth than a short. When its phase is well known, the reflect can be used to define the electrical reference plane instead of the THRU. In the present case we used the open as the reflect standard.

\subsubsection{Line}

The one precision standard is the Line. In some cases, such as zero length THRU, its characteristic impedance $Z_{0}$ sets the reference impedance for the entire $\mathrm{RF}$ test system. For good RF measurements, the line's impedance should be precisely defined, otherwise, normalization is required. TRL sets the test system's $Z_{0}$ to be equal to the impedance of the line standard. However, tolerance in the conductor etch fabrication can result in the line's impedance being slightly different than $Z_{0}$. Since the DUT measurements are defined in terms of $Z_{0}$, error will result.

Line loss and dispersion will impact the impedance of the line's $Z_{0}$. The loss in the line standard is partly due to the metal's conductivity, which is a function

of the skin depth. Making assumptions about transmission line symmetry can sometimes compensate for calibration errors due to line loss [10]. 


\subsection{TRL line design}

Complete microstrip models indicate that the physical length and electrical length are related not only by the dielectric constant, but also the thickness of the dielectric, and the dimensions and conductivity of the surface and ground conductors.

However, precise specification of the electrical length is not required in TRL, particularly when a non-zero-length THRU is used to set the reference plane.

\subsection{Selecting the optimal THRU and Line lengths}

According to the design example provided by Agilent [2], for PC board applications, they recommend using non-zero-length THRU $=20 \mathrm{~mm}$. Additionally, a nominal open is preferred rather than a short circuit.

Therefore, we set the electrical length of the THRU to $20 \mathrm{~mm}$. The physical length of the THRU can be estimated as follows.

$$
\text { Physical length }=\frac{\text { Electrical length }}{\sqrt{\epsilon_{r}}}
$$

Thus, the physical length of the THRU turns out to be equal to $9.53 \mathrm{~mm}$ for electrical length of $20 \mathrm{~mm}$ and dielectric constant of FR4 to be equal to 4.4. For the designed calibration kit the THRU's length was $9.116 \mathrm{~mm}$. This value of line length was calculated by LineCalc software in ADS when the appropriate substrate and line width were specified.

Assuming a transmission media that exhibits linear phase, the following expression can be used to select a line with $1 / 4$ wavelength line at the center frequency.

$$
l=\frac{1}{4} \lambda \ldots \text { at center frequency }
$$




$$
v=\lambda f
$$

where $v$ is called the phase speed (magnitude of the phase velocity) of the wave and $f$ is the wave's frequency. In the case of electromagnetic radiation, such as light in free space, the phase speed is the speed of light c.

$$
\lambda=\frac{c}{f}
$$

where,

$$
\begin{gathered}
c=3 \times 10^{11} \ldots \text { speed of light in } \frac{m m}{s} \\
f_{\text {center }}=\frac{f_{1}(G H z)+f_{2}(G H z)}{2}
\end{gathered}
$$

In eq. 3.2 , we can substitute the values of $\mathrm{c}$ and $f_{\text {center }}$.

This gives us,

$$
l(m m)=\frac{300}{4} \times \frac{2}{f_{1}(G H z)+f_{2}(G H z)}
$$

The electrical length can be related to physical length when the effective permittivity of the dielectric is known. In designing these lines we have used the effective permittivity of dielectric $\epsilon_{r}=4.4$

$$
\text { Electrical length } l(m m)=\frac{150}{f_{1}(G H z)+f_{2}(G H z)}
$$

Also, 


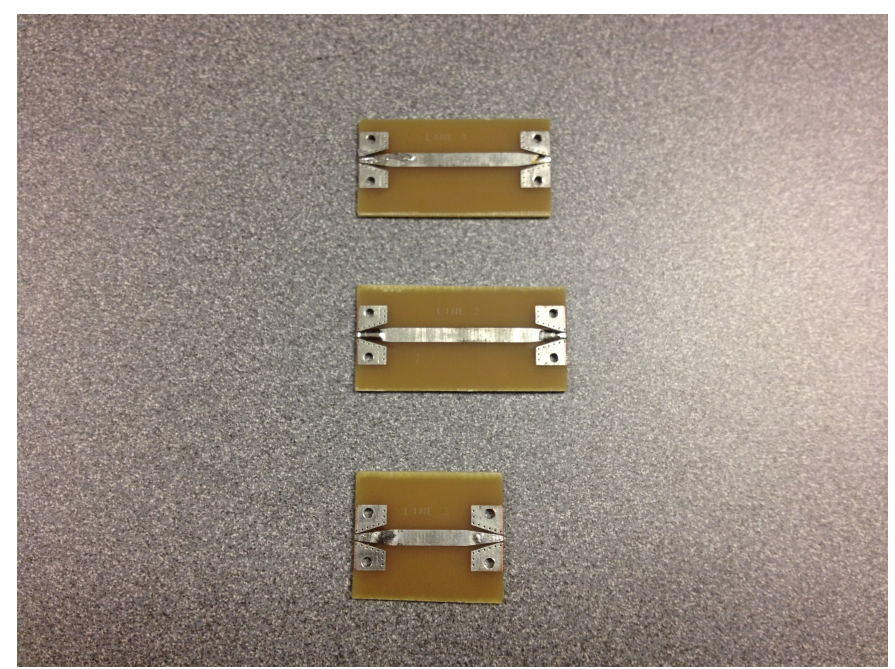

Figure 3.2: Calibration Lines

$$
\text { Electrical length } l(m m)=L I N E-T H R U
$$

In our case, $f_{1}(G H z)=0.5 \mathrm{GHz}$ and $f_{2}(G H z)=10 \mathrm{GHz}$

According to the TRL requirements in [2] the desired frequency span must be divided, allowing one $1 / 4$ wavelength line to be used over the lower portion of the frequency span and a second to be used for the upper band. The optimal break frequency is the geometric mean frequency.

$$
\sqrt{f_{1} \times f_{2}}
$$

The frequency range we are examining is $0.5 \mathrm{GHz}$ to $10 \mathrm{GHz}$, so the optimal break frequency is $2.236 \mathrm{GHz}$. For the purposes of overlapping of the frequency bands we will be using two ranges, one from $0.5 \mathrm{GHz}$ to $3 \mathrm{GHz}$ and the other from $3 \mathrm{GHz}$ to $10 \mathrm{GHz}$. We added one more line to this so as to add to the functionality of the calibration kit, ranging from $0.5 \mathrm{GHz}$ to $3.5 \mathrm{GHz}$.

This gives us three electrical lengths shown in Figure 3.2. 


\begin{tabular}{|c|c|}
\hline Frequency $(\mathrm{GHz})$ & Phase (degrees) \\
\hline \multicolumn{2}{|c|}{ Line $137.5 \mathrm{~mm}$} \\
\hline 0.5 & 22.5 \\
\hline 3.5 & 157.5 \\
\hline \multicolumn{2}{|c|}{ Line $242.85 \mathrm{~mm}$} \\
\hline 0.5 & 25.2 \\
\hline 3 & 154.26 \\
\hline \multicolumn{2}{|c|}{ Line $312.5 \mathrm{~mm}$} \\
\hline 2 & 30 \\
\hline 10 & 150 \\
\hline
\end{tabular}

Table 3.1: Phase relationship of lines

1. LINE $1_{E}=37.5 \mathrm{~mm}$, frequency range $0.5 \mathrm{GHz}$ to $3.5 \mathrm{GHz}$

The physical length of the LINE $1_{P}=17.851 \mathrm{~mm}$. Adding this to the THRU length gives us the final value of LINE $1=(17.851+9.117)=26.968 \mathrm{~mm}$.

2. LINE $2_{E}=42.85 \mathrm{~mm}$, frequency range $0.5 \mathrm{GHz}$ to $3 \mathrm{GHz}$

The physical length of the LINE $2_{P}=20.427 \mathrm{~mm}$. Adding this to the THRU length gives us the final value of LINE $2=(20.427+9.117)=29.544 \mathrm{~mm}$.

3. LINE $3_{E}=12.5 \mathrm{~mm}$, frequency range $2 \mathrm{GHz}$ to $10 \mathrm{GHz}$

The physical length of the LINE $3_{P}=5.959 \mathrm{~mm}$. Adding this to the THRU length gives us the final value of LINE $3=(5.959+9.117)=15.076 \mathrm{~mm}$.

According to the TRL requirements in [2] the insertion phase difference between the THRU and line must be between (20 and 160 degrees) $\pm \mathrm{n} \times 180$ degrees. To determine whether this line meets the conditions of acceptable insertion phase, the following expression was used:

$$
\text { Phase (degrees })=\frac{360 f l}{c}=12 f(G H z) l(\mathrm{~cm})
$$




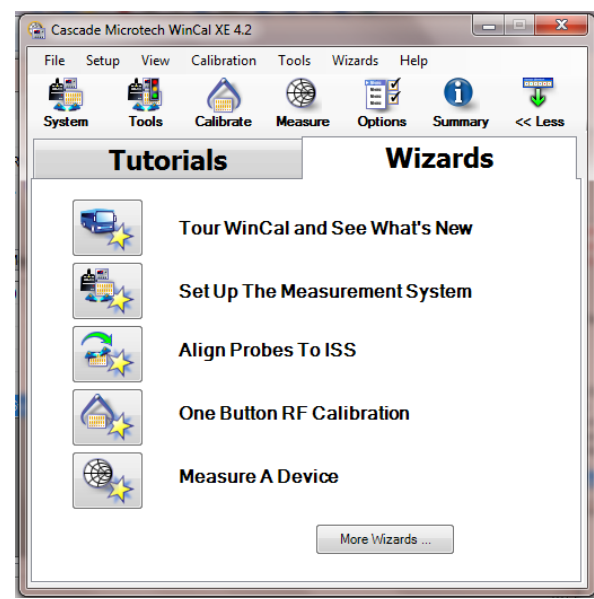

Figure 3.3: WinCal's Main Window

Table 3.1 shows the phase calculation for the three lines that were used. All three lines satisfy the phase criterion. In order to cover greater than an 8:1 frequency span, multiple lines must be used.

In the next section, there is a short discussion about use of the Cascade Microtech's WinCal XE software which was used for calibrating the data.

\subsection{Cascade Microtech's WinCal XE calibration process}

Here is WINCAL's stepwise procedure for performing the TRL calibration

- As soon as you start WinCal XE Figure 3.3 window will show. This is the main page of WinCal XE software.

- The first step is to Setup the System

In this case we used the Virtual VNA. Click Confirm Communication. This will give some information of the port that it is connected to. Use VNA Settings tab for selecting the error terms. Select the ideal error terms. Go to Stimulus Settings. See Figure 3.4. 


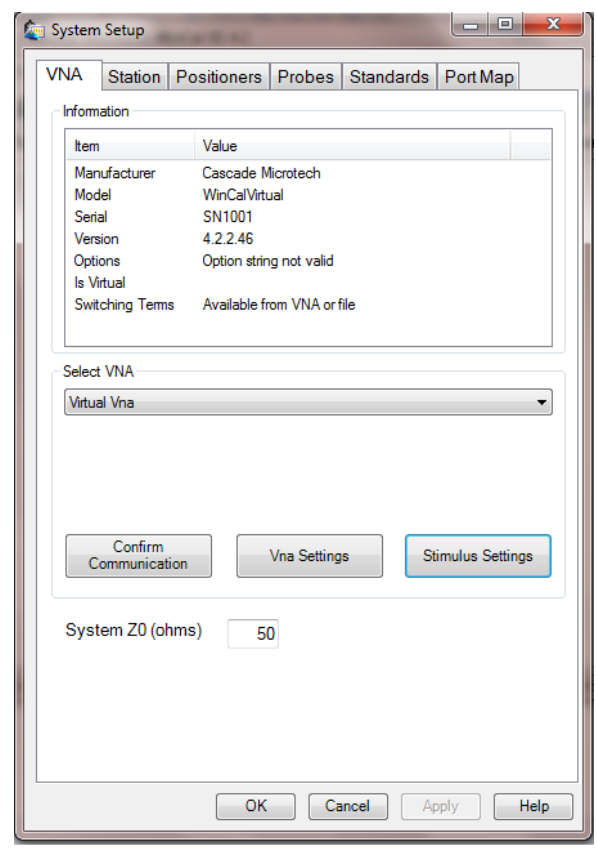

Figure 3.4: WinCal's Setup Window

- Virtual VNA stimulus Settings. See Figure 3.5.

$\rightarrow$ Select the Frequency List as per your RAW data, either Segment or Linear.

$\rightarrow$ Source File, import the raw data on which calibration has to be applied.

$\rightarrow$ Select the File Name to which you want to apply the calibration.

$\rightarrow$ File Sweep Mode Select "Direct From File" and the File Freq Range will be selected accordingly. After you make the changes click Apply in the System Setup Window and then click $O K$.

- Go to the Main Window and click Calibrate. The Calibration window will pop up as shown in the Figure 3.6.

Select the Calibration Algorithm that you want to use. In our case we used the 2-port TRL calibration. Then go to Setup and a new window will be opened as shown in Figure 3.7. 


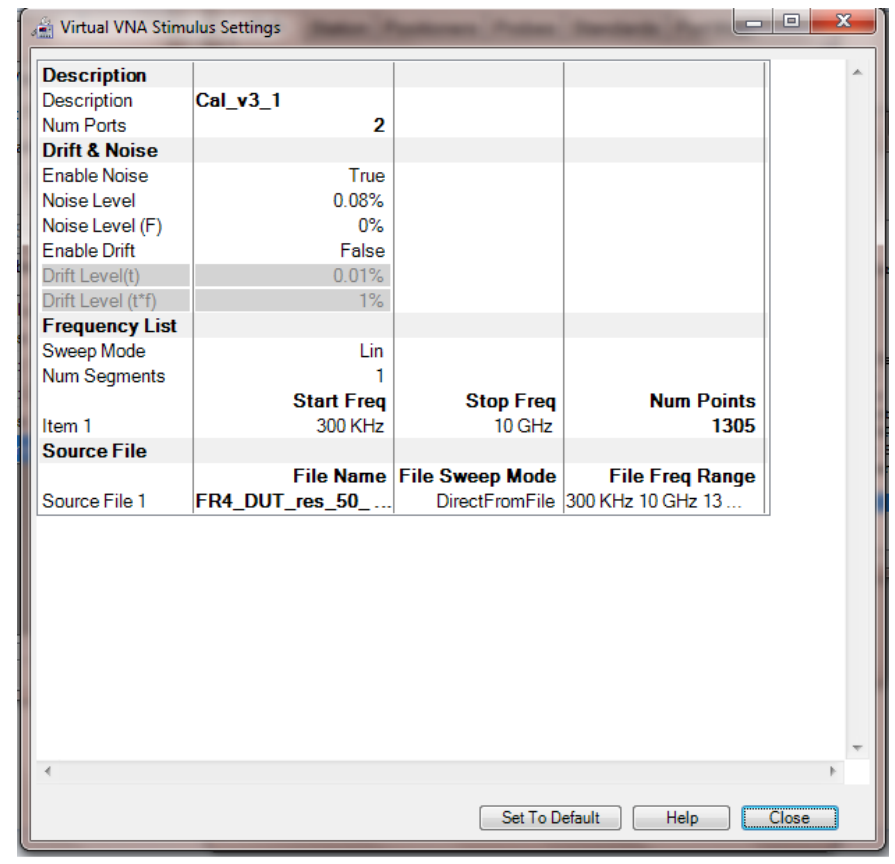

Figure 3.5: VNA Stimulus Settings

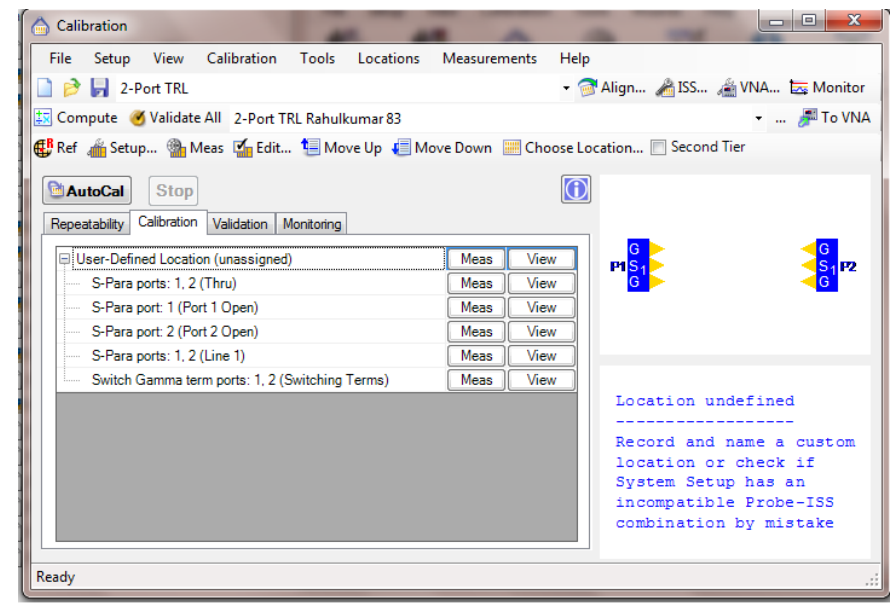

Figure 3.6: Calibration Window 


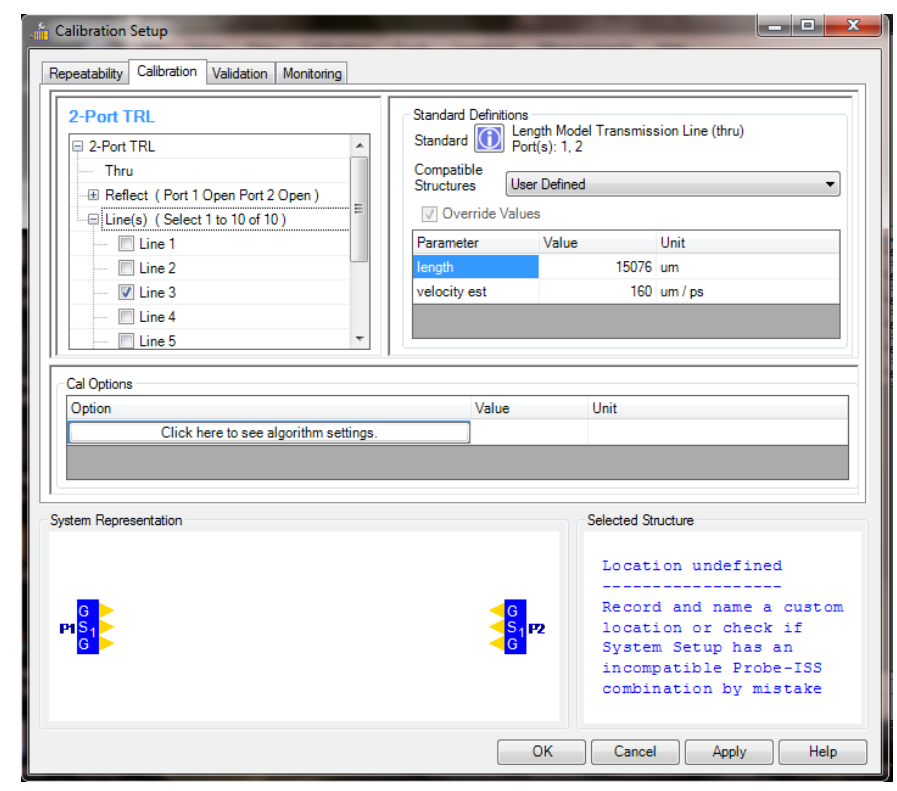

Figure 3.7: Calibration Setup Window

- Calibration Setup

Figure 3.7 will be opened. Add the details of the lines and the THRU used. Add the correct lengths of lines in the Value section. For example, if we select line 3 in the Lines section of the Calibration setup then we have to use the length of line 3 as specified in the previous section, which is $15.076 \mathrm{~mm}$. Similarly depending on the line used, select the appropriate length of line. For TRL add the range of frequencies of lines used.

- TRL settings in Figure 3.8. Select the range of frequencies for the lines used.

- Click AutoCal and after it goes through all the process load the measured data by right clicking on the respective standard to load the raw data from a measurement file. After the data is loaded click Compute so that the error set is calculated depending on the current data (i.e. the THRU and the line).

- Go to the main window and click Measure (see Figure 3.9). 


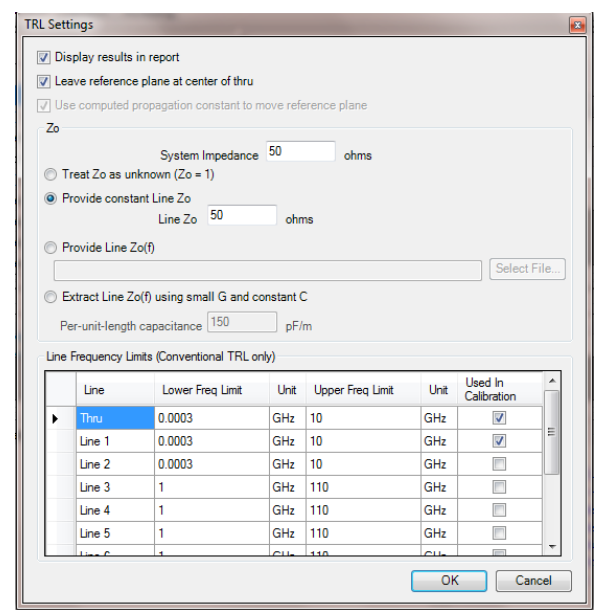

Figure 3.8: TRL calibration settings

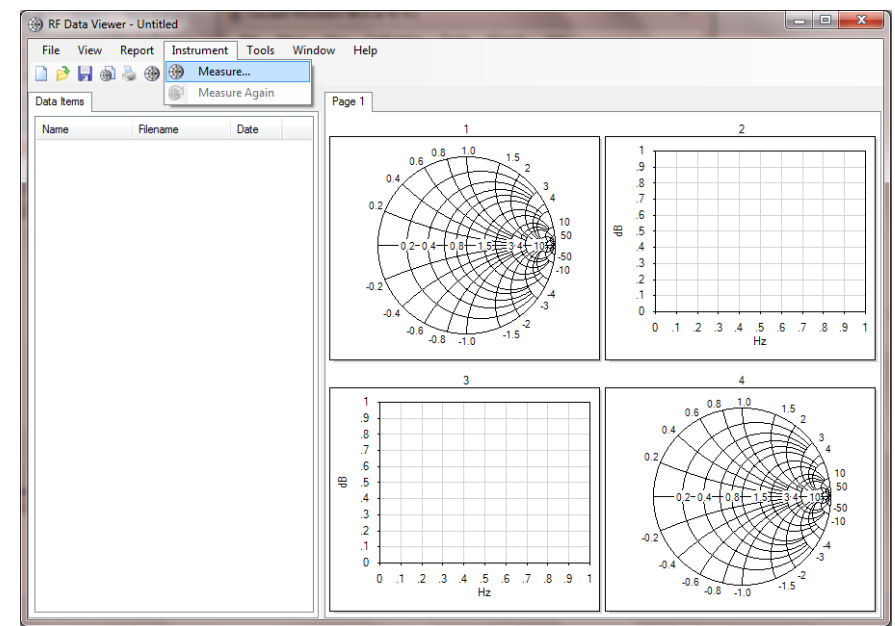

Figure 3.9: Measurement Window

The raw data which was included in the source file will be calibrated and the new calibrated data will be plotted in this new measure window.

- Saving this measured data

In order to save this calibrated data, right click on the file and there will be an option to Export the file. Export this file to the desired folder and the touchstone file will be saved in the destination folder. This .s2p file can be read by Agilent's ADS for further data processing. 


\section{Chapter 4}

\section{Measurement and Modeling}

\subsection{Measurement Setup}

In order to validate the measured and modeled data it is necessary to compare this data with a well known measurement such as LCR meter data. The following procedure was adhered to for performing the measurement.

1. Measure the SMD component using LCR meter (Agilent E4980A).

Each component was measured at 201 frequency points between $20 \mathrm{~Hz}$ and

$2 \mathrm{MHz}$. Depending on the type of the component we will change the measurements taken by the LCR meter. The LCR meter has many functions with which we can measure the parasitic series resistance, shunt capacitance, series inductance, etc.

- For the resistor we take the following measurements

Impedance $(\mathrm{Z}) \leftrightarrow$ angle in degrees $\left(\theta_{d}\right)$ and

series inductance $\left(L_{s}\right) \leftrightarrow$ series resistance $\left(R_{s}\right)$

- Similarly, for the capacitor we take the measurements of Impedance $(\mathrm{Z}) \leftrightarrow$ angle in degrees $\left(\theta_{d}\right)$ and series capacitance $\left(C_{s}\right) \leftrightarrow$ series resistance $\left(R_{s}\right)$

- For the inductor we take Impedance $(\mathrm{Z}) \leftrightarrow$ angle in degrees $\left(\theta_{d}\right)$ and series inductance $\left(L_{s}\right) \leftrightarrow$ series resistance $\left(R_{s}\right)$ 
These measurements are taken by changing the appropriate function on the LCR meter each time before measurement.

2. Solder the SMD component on the SMD pad/site. Pad size recommended by SMD manufacturer is used. Ensure the connectivity of the component solder by connecting it to the high precision digital multimeter $34410 \mathrm{~A}$ by Agilent.

3. The Tektronix's DSA8200 Digital Serial Analyzer is used to measure the impedance of the microstrip transmission lines.

4. Vector Network Analyzer (VNA) is used to measure the S-parameters of the resistors, capacitors and inductors. Before taking the measurements on the VNA calibrate it using the Electronic Calibration Kit (ECAL) provided by Agilent. This will calibrate the VNA up to the SMA (Sub-miniature A) cable tips. Adjust the frequency range of the VNA so that we can take some measurement in the lower frequency region which can be used later for comparison with the LCR meter (for example, in the frequency range from $300 \mathrm{KHz}$ to $2 \mathrm{GHz}$ take 50 measurements). Change the IFBW (Intermediate Frequency Bandwidth) on the VNA to $1 \mathrm{KHz}$ so as to get a good dynamic range of measurement. Measure the S-parameters of the SMD components and save the data in touchstone file format (.s2p format).

5. This is the raw data which we have to import in Cascade Microtech's WinCal XE software. It is used for extensive calibration and repetitive S-parameter measurement. This software will apply the TRL algorithm to this raw data so we can obtain the calibrated data which is the actual measurement of the SMD component. Refer to the block diagram shown in Figure 4.1. 


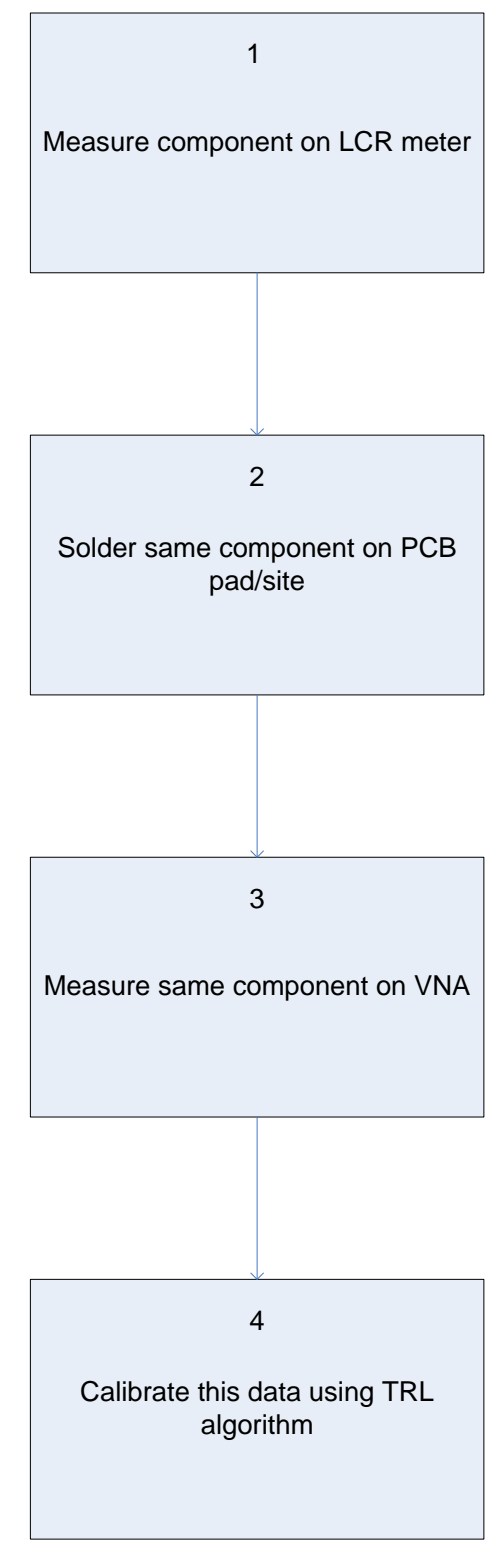

Figure 4.1: Measurement Setup 1 
6. Import newly calibrated data in ADS so that we can apply extensive optimization and fit the simple model to the measured data. Three optimization algorithms were applied to get closer to measured data. Convert these Sparameters to Y-parameters so that we can compute the pad capacitance as shown in the model in Figure 2.7.

7. Compute the impedance from the Y-parameters and compare it with the data obtained from the LCR meter. This can be done by taking the inverse of the Y12 parameter. This is the impedance of the component. This is the final value to be compared for the resistor, but for the capacitor and the inductor this is the capacitive reactance and inductive reactance. Using these values we have to extract the capacitance and inductance respectively.

8. Put this data on graph for comparison purposes and this will show how good the simple model is versus the actual model given by the manufacturer. Repeat this procedure for the same values of resistors, capacitors and inductors to verify the validity of the data. Refer to Figure 4.2 .

Block diagrams of the entire measurement process are shown in Figure 4.1 and Figure 4.2.

\subsection{Model extraction setup - Optimization description}

ADS gives us the option of optimizing the design so as to achieve the best results of modeled circuits [5].

Overall, optimization is the process of modifying a set of parameter values to satisfy performance goals. Optimizers compare VNA measured data and desired 
5

Optmize Calibrated data in ADS

6

Compare Optimized, measured and nominal data

7

Compute impedance and compare to LCR meter data

8

Repeat this process and compare the data for same values of different components

Figure 4.2: Measurement Setup 2 
responses (our modeled data which is supposed to be comparable to the VNA measured data) and modify design parameter values to bring the computed response closer to the desired.

The steps of nominal optimization are as follows:

1. Running a simulation (in our case we are importing the measured data using the Data Access Component (DAC) and calculating the required parameters).

2. Comparing results with the goal.

3. Changing the circuit parameters to obtain results that are likely to be closer to the goal.

4. Running the simulation again with the new parameter values.

Minimum requirements for defining an optimization process in ADS are:

- Goals are the specifications for the circuit performance to be met.

- Optimization variables/parameters are the critical design parameters, to which the circuit performances are sensitive.

- Optimization methodology and optimizers define the approach to adjust the optimization variables/parameters to meet the goals.

Every optimizer uses two functions.

1. Error function formulation

2. Search Methods

Three different Optimizers were used: Random, Simulated Annealing and Gradient 


\subsubsection{Random Optimizer}

The Random optimizer uses the random search method to arrive at the new parameter values by using a random-number generator, that is, by placing a number at random within a range.

The Random optimizer uses the least-squares error function to minimize the average weighted violation for the desired responses. So the value of the error function represents the average weighted violation for the desired responses and the value of zero indicates that all of the intended performance goals have been reached.

\subsubsection{Simulated Annealing}

Simulated Annealing is a technique that has attracted significant attention as a suitable choice for optimization problems of large scale, especially ones where a desired global extremum is hidden among many, poorer, local extrema.

Simulated Annealing can deal with highly nonlinear models, chaotic and noisy data and many constraints. It is a robust and general technique, its main advantages over other local search methods are its flexibility and its ability to approach global optimality. However, a lot of choices are required to tune it for an actual problem. There is a clear trade-off between the quality of the solutions and the time required to compute them. The tailoring work required to account for different classes of constraints and to fine-tune the algorithm's parameters can be rather delicate. We had to fine tune parameters like the shunt capacitances and series inductance so that these simple models match the measured data. 


\subsubsection{Gradient Optimizer}

The Gradient Optimizer uses the Gradient search method to arrive at the new parameter values using the gradient information of the network's error function. The gradient of the error function indicates the direction to move a set of parameter values in order to reduce the error function.

It uses the same error function as used by the Random Optimizer. A design that is optimized by the gradient optimizer has the least sensitivity (i.e. it is more stable) to slight variations in its parameter values.

\subsection{Optimization Description}

Extensive optimization was performed so as to fit the measured data to the modeled data. Simple models consisting of small series resistance and shunt capacitance were modeled so as to simulate the behavior of the real world passive component in ADS simulation software. The calibration process sets the reference plane to the end of the SMD pads but while simulating the models of these components the pad capacitance was considered in order to take into account their parasitics.

There were seven goals for which three optimizers were used to get to the optimal value. Out of these seven goals, four were used for the S-parameters and the remaining three were used for the Y-parameters in the reference model, see Figure 2.7. Three optimizers were used because by cascading multiple optimizers we obtained better results as compared to using a single optimization technique.

The gradient optimizer in some cases did not give us better results but in some cases it gave comparable results. Removing this optimizer could cause some of the dependent results to loose the accuracy so it was kept so as to maintain consistency throughout the optimization process. 


\begin{tabular}{|c|c|c|c|c|}
\hline Resistor & $22 \Omega$ & $34 \Omega$ & $56 \Omega$ & $75 \Omega$ \\
\hline Capacitor & $1.5 \mathrm{pF}$ & $3.3 \mathrm{pF}$ & $15 \mathrm{pF}$ & $33 \mathrm{pF}$ \\
\hline Inductor & $2.2 \mathrm{nH}$ & $22 \mathrm{nH}$ & $22 \mu \mathrm{H}$ & \\
\hline \hline
\end{tabular}

Table 4.1: Component selection

Figure 4.3 shows an example of the optimization performed in ADS.

\subsection{Component Selection}

Four different values of the resistors and capacitors were measured, along with three values of inductors. For each of the SMD components, three samples for each value were measured to get a sense of variability of the component values.

Table 4.1 shows the values of the resistors, capacitors and inductors.

\subsection{Resistor Modeling}

The resistor used was a high frequency resistor, thin film surface mount chip - 0603 size from Susumu Corporation Limited. It was measured using Agilent's ENA series Network Analyzer. The measured data was calibrated using the WinCal XE software and then optimized in ADS. A model was created in ADS according to the design given by the Vishay semiconductor datasheet, because Susumu Corporation's model was not available. This model was used as a reference to check the functionality of the simple model developed by us.

Measured S parameters of $22 \Omega$ resistor were plotted in Figure 4.4. The graph shows the results of the modeled data (in red), measured data (in blue) and nominal data (in black).

From the graph we can see that the measured and the modeled data match each other very closely and so the model used approximates the $22 \Omega$ resistor very 


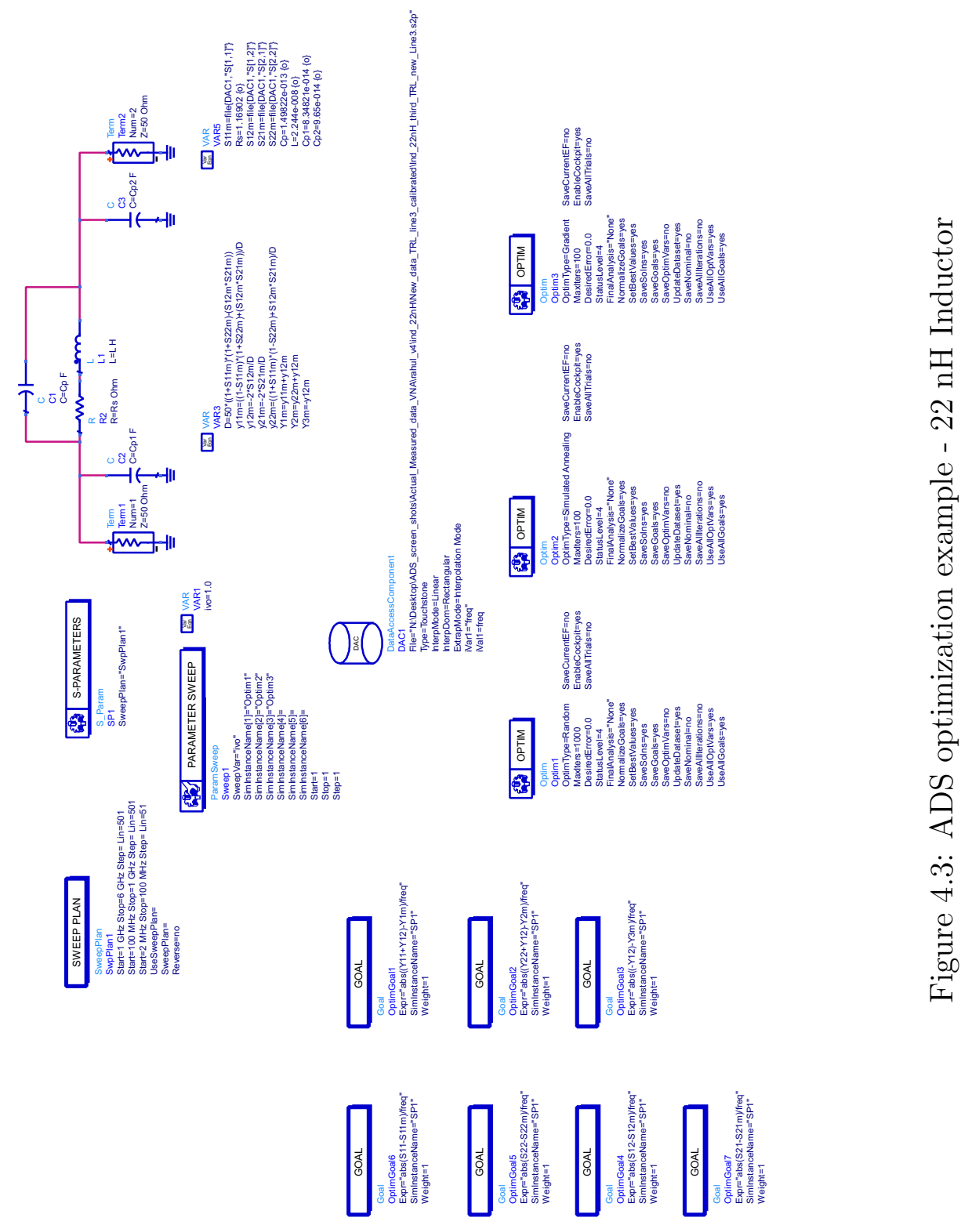


well. This also verifies that the calibration algorithm used is working properly.

To further verify whether the measured component was actually a resistor we used the Smith chart to get a better visualization of the measured component. From the Figure 4.5 we can see that the measured component indeed behaves as a resistor with some series inductance.

\subsection{Capacitor Modeling}

The capacitor used was GQM1885C1H330JB01D. It is a $33 \mathrm{pF} 0603$ SMD capacitor by Murata Manufacturing Co. Limited. The tolerance of the capacitor is $\pm 5 \%$ and voltage rating is $50 \mathrm{~V}$. The capacitor was first measured and then compared to the model which was provided by Murata as a part of ADS component library.

Measured S parameters of $33 \mathrm{pF}$ capacitor were plotted in ADS. The graph in Figure 4.6 shows the results of the modeled (in red), measured (in blue) and nominal data (in black) after optimization.

To verify whether the measured component was actually a capacitor we used the Smith chart to get a better visualization of the measured component. From the Figure 4.7 we can see that the measured component is indeed a capacitor.

\subsection{Inductor Modeling}

The inductor model was developed based on the considerations in Impedance Handbook [4].

The inductor that was used for the measurement was Murata RF inductor -

LQP18MN22NG02. It is a 22nH chip coil $0603 \mathrm{SMD}$ inductor with $\pm 2 \%$ tolerance. Murata provided the ADS model for this inductor as well.

Measured S parameters of $22 \mathrm{nH}$ inductor were plotted in ADS. The Figure 4.8 


\begin{tabular}{|cc|}
\hline$d B(S(1,1))$ & \\
$d B(S 11 m)$ & $=-0=$ \\
$d B($ resistor_22_Vishay_model..S(1,1)) & \\
\hline
\end{tabular}
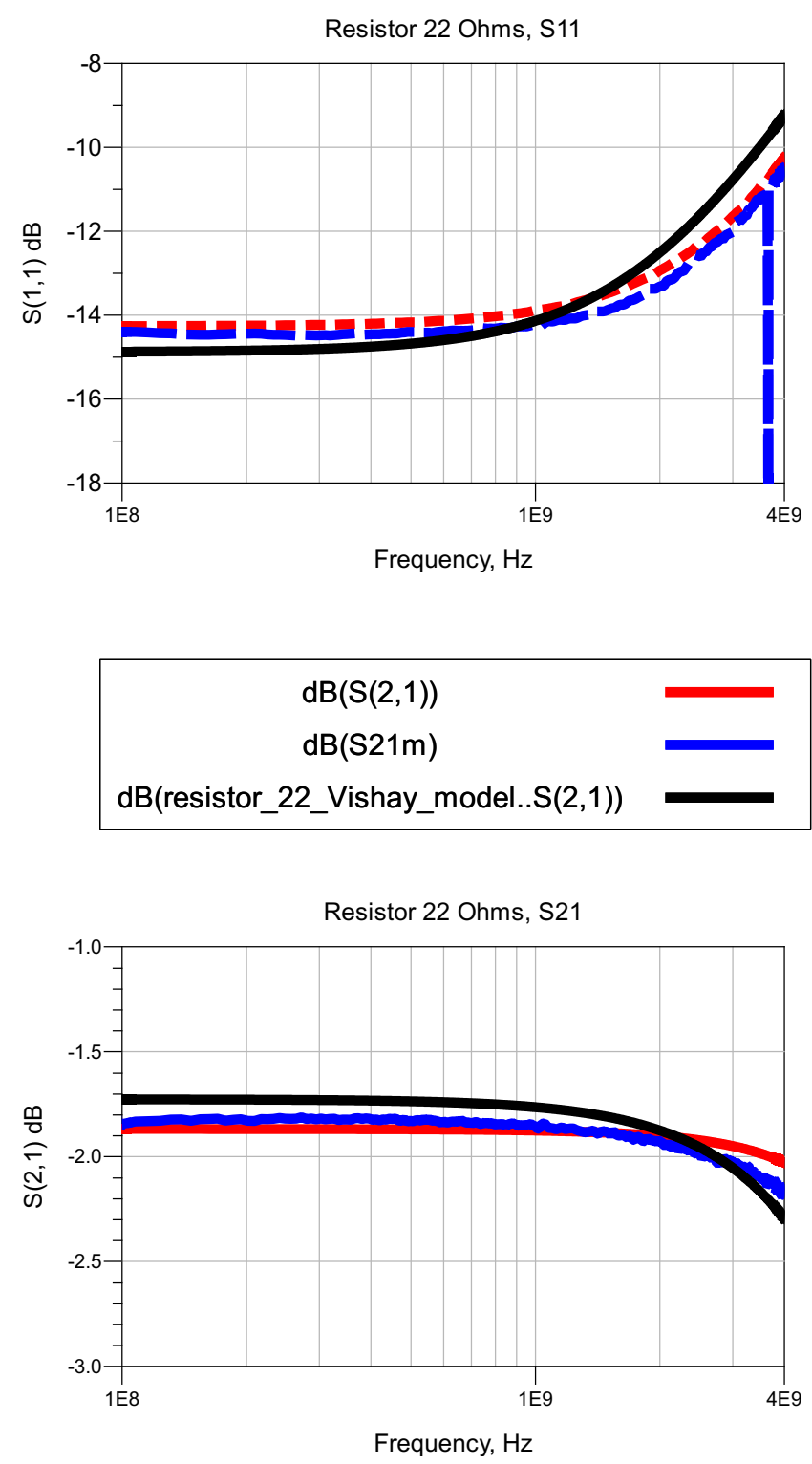

Figure 4.4: Comparison of extracted model (red), measured component (blue) and nominal model (black) values for S11 and S21 of $22 \Omega$ resistor. 


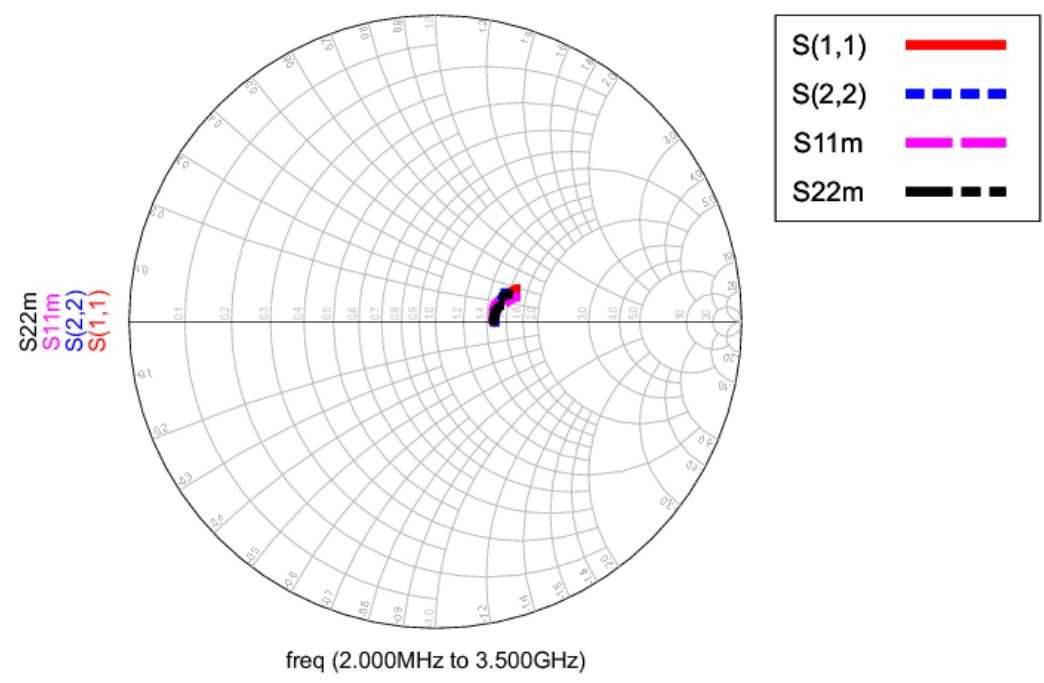

Figure 4.5: Smith chart illustration of frequency dependence of S11 and S22 for measured component (pink and black) and extracted model (red and blue) for 22 $\Omega$ resistor.

shows the results of the modeled (in red), measured (in blue) and nominal data (in black) after optimization.

Due to the surface contact pads used by $2.2 \mathrm{nH}$ and $22 \mathrm{nH}$ chip coil, it was difficult to measure these components on the LCR meter due to improper contacts. Therefore, for verifying the inductor model we used an inductor of $22 \mu \mathrm{H}$ which had side pads so that it could make contact with the LCR meter's measuring test fixture (Agilent 16034E Test fixture). The $22 \mu \mathrm{H}$ inductor is of 0805 size.

To verify whether the measured component was actually a inductor we used the Smith chart to get a better visualization of the measured component. From the Figure 4.9 we can see that the measured component is indeed a inductor. 

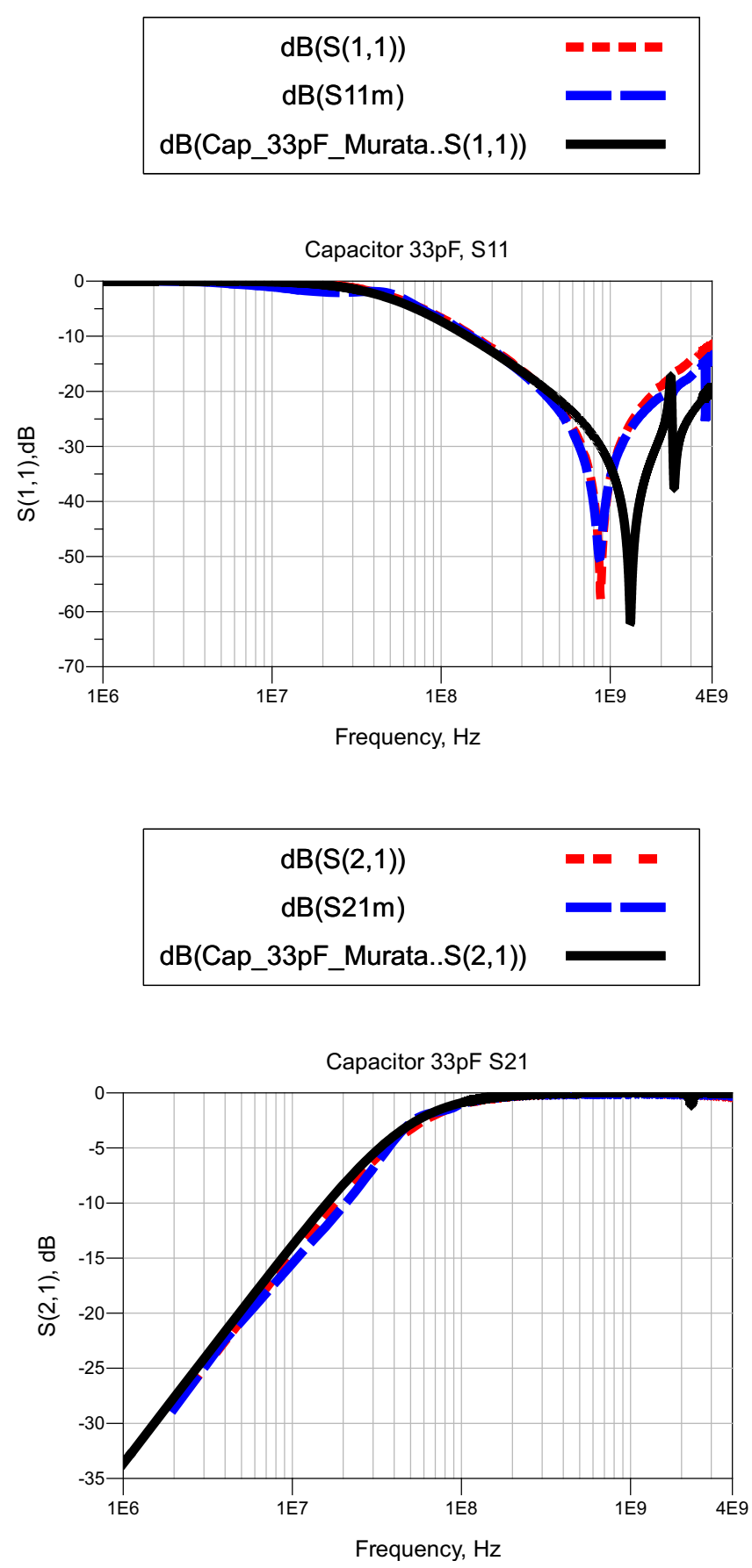

Figure 4.6: Comparison of extracted model (red), measured component (blue) and nominal model (black) values for S11 and S21 of 33 pF capacitor. 


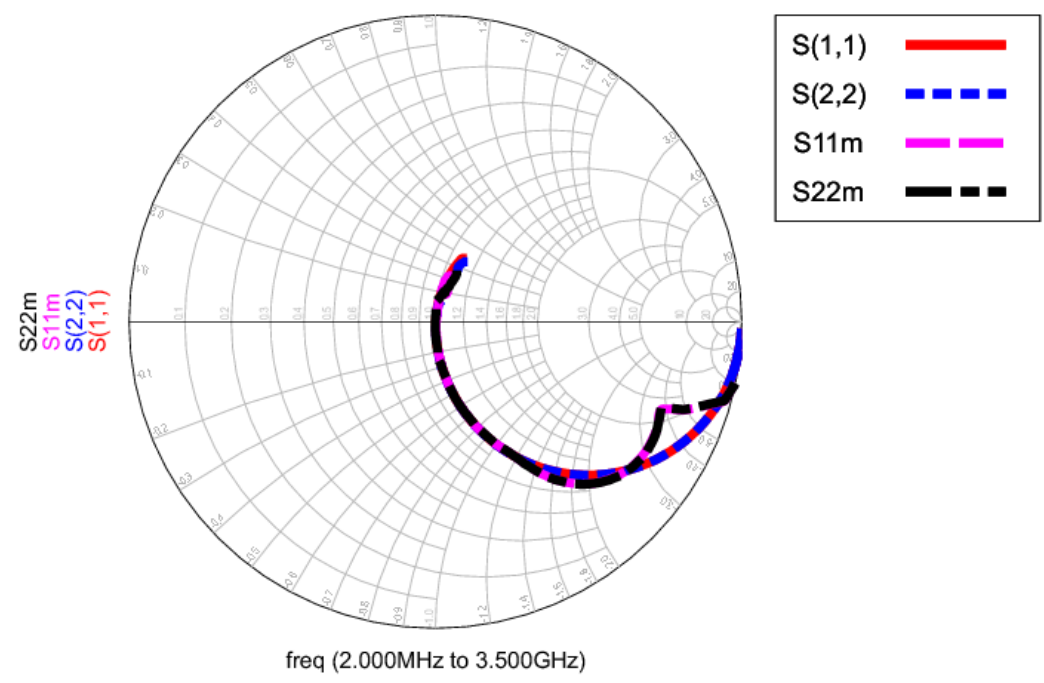

Figure 4.7: Smith chart illustration of frequency dependence of S11 and S22 for measured component (pink and black) and extracted model (red and blue) for 33 $\mathrm{pF}$ capacitor.

\subsection{Measurement Observations}

From the S-parameters that were plotted we observed that S11 and S22 are the same, as are S12 and S21, so that device is reciprocal. This is because in our case we are using the same type of connector and fixture at port 1 and port 2 so they look similar. This is evident from the Smith charts of resistor, capacitor and inductors. The phase of these S-parameters looks as expected.

We were able to model the pad capacitances in a very satisfactory manner. The Cpad makes small contributions to the design and does not affect performance of the system to a great extent. From the values that are obtained from the Cpad, we conclude that the value of the Cpad is consistent across the group of components of the same type. For example, value of the Cpad is consistent for most of the values of the resistors and is in the range of $0.01 \mathrm{pF}$. On the other hand, the 


\begin{tabular}{|cc|}
\hline$d B(S(1,1))$ & $=-=-$ \\
$d B(S 11 m)$ & $=$ \\
$d B($ Ind_22nH_Murata..S(1,1)) & $=$ \\
\hline
\end{tabular}
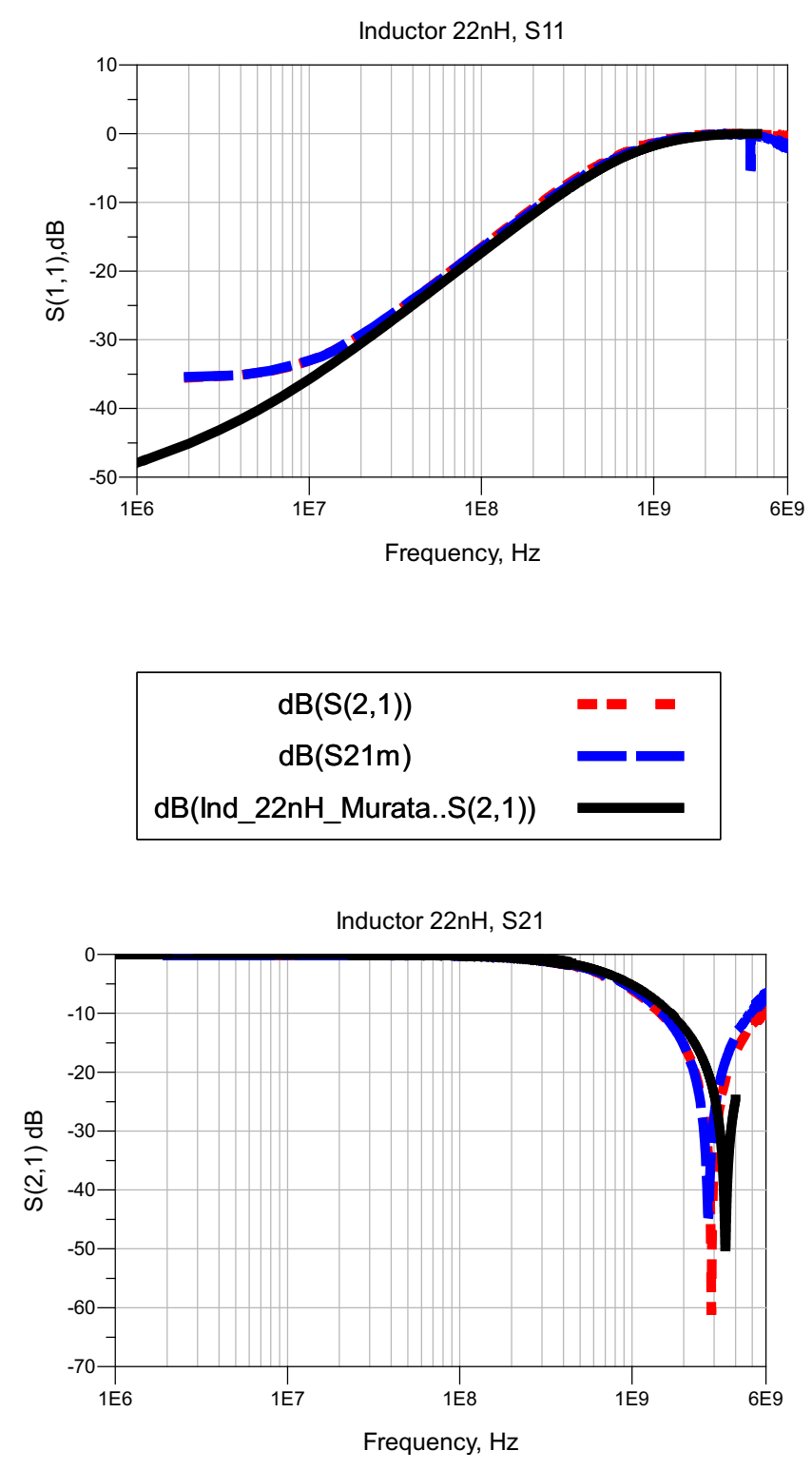

Figure 4.8: Comparison of extracted model (red), measured component (blue) and nominal model (black) values for S11 and S21 of $22 \mathrm{nH}$ inductor. 


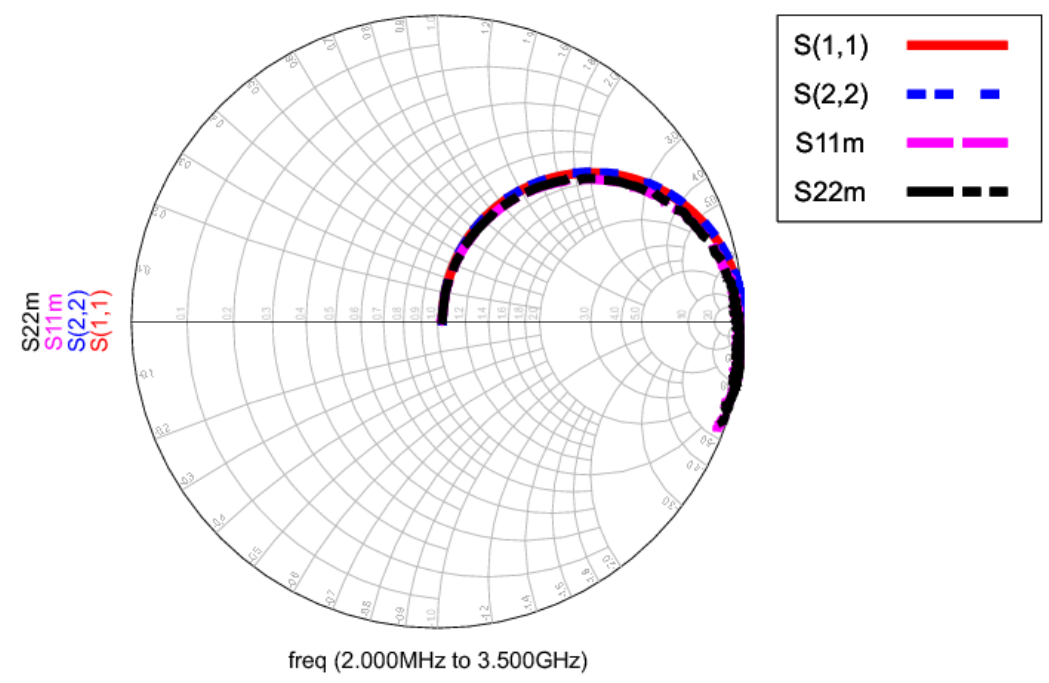

Figure 4.9: Smith chart illustration of frequency dependence of S11 and S22 for measured component (pink and black) and extracted model (red and blue) for 22 $\mathrm{nH}$ inductor.

pad capacitance in the case of inductors and capacitors is in the range of 0.5 $\mathrm{pF}$ to $0.9 \mathrm{pF}$. The pad capacitance in case of " $\mathrm{L}$ " and "C" not only takes into consideration its pad capacitance but also shows some parasitic components of the actual measured inductor or capacitor.

The parasitic inductance $L_{s}$ in case of capacitor and shunt capacitance $C_{p}$ in case of inductor decide the resonant frequency of the component. 


\section{Chapter 5}

\section{Summary of Results and Discussion}

\subsection{Comparison of measured and modeled data - Resistor, Capacitor and Inductor}

The passive surface mount devices were first measured using Agilent's E4980A Precision LCR meter. The operating frequency range of this instrument is from $20 \mathrm{~Hz}$ to $2 \mathrm{MHz}$. This LCR meter was used to verify the actual value of the components as opposed to nominal value specified by the component manufacturer.

Agilent's E5071C ENA series Network Analyzer was also used for performing measurements in the $300 \mathrm{KHz}$ to $10 \mathrm{GHz}$ range. For VNA calibration we used an Agilent N4619B electronic calibration kit. This type of electronic calibration kit is beneficial because it can calibrate VNA to the end of SMA cables without the operator having to define their own calibration standards and is repeatable as well as accurate.

\subsection{Resistor Results}

Table 5.1 shows the results of modeled data after extensive optimization was performed in ADS.

From Table 5.1 we can observe that the optimized modeled resistor values are in close proximity to each other. From the results presented in Figure 4.4 and similar results for other resistors we know that modeled S parameters follow the measured S parameters closely. A full set of plots comparing modeled and measured results is given in the Appendix. 


\begin{tabular}{|l|l|l|l|l|l|}
\hline Resistor $(\Omega)$ & $\operatorname{Rs}(\Omega)$ & $\operatorname{Ls}(\mathrm{nH})$ & $\operatorname{Cr}(\mathrm{pF})$ & $\operatorname{Cpad}(\mathrm{pF})$ & $\operatorname{Cpad}(\mathrm{pF})$ \\
\hline \multicolumn{7}{|c|}{ Resistor $22 \Omega$} \\
\hline 23.24 & 0.49 & 2.61 & 0.18 & 0.15 & 0.15 \\
\hline 24.19 & 0.19 & 2.49 & 0.10 & 0.16 & 0.14 \\
\hline 23.50 & 0.49 & 0.20 & 0.53 & 0.12 & 0.10 \\
\hline \multicolumn{7}{|c|}{ Resistor $34 \Omega$} \\
\hline 35.8 & 0.50 & 2.93 & 0.10 & 0.16 & 0.16 \\
\hline 35 & 0.49 & 2.49 & 0.10 & 0.06 & 0.12 \\
\hline 34.5 & 0.39 & 2.45 & 0.12 & 0.12 & 0.17 \\
\hline \multicolumn{7}{|c|}{ Resistor $56 \Omega$} \\
\hline 61.46 & 0.05 & 3.93 & 0.19 & 0.18 & 0.19 \\
\hline 58.8 & 0.39 & 3.68 & 0.17 & 0.17 & 0.18 \\
\hline 61.54 & 0.04 & 4.01 & 0.25 & 0.18 & 0.19 \\
\hline \multicolumn{7}{|c|}{ Resistor $75 \Omega$} \\
\hline 78.75 & 0.39 & 4.42 & 0.18 & 0.20 & 0.21 \\
\hline 82.5 & 0.29 & 4.73 & 0.17 & 0.21 & 0.22 \\
\hline 69.98 & 0.09 & 4.12 & 0.11 & 0.21 \\
\hline \hline
\end{tabular}

Table 5.1: Resistor model parameters for a given nominal component value. Measurements done on VNA.

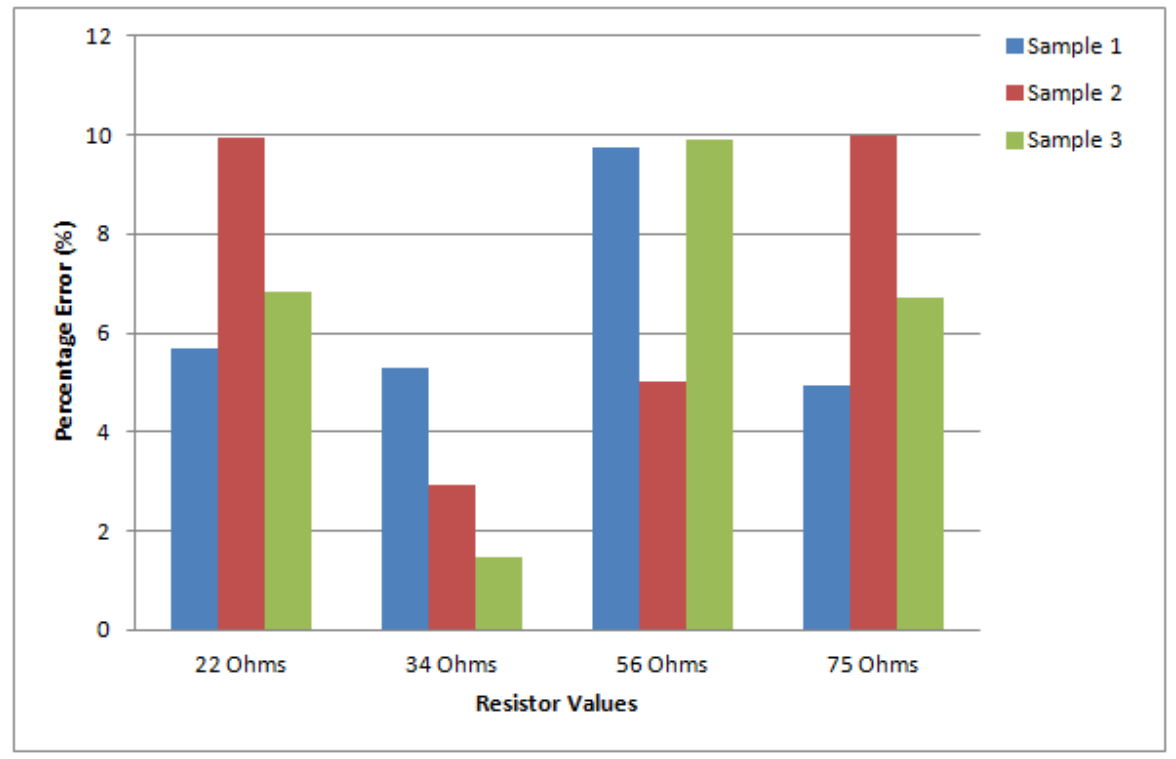

Figure 5.1: Resistor percentage error with respect to nominal value for three samples. 
The pad capacitances for Pad1 and Pad2 for all the resistors are very close to each other. This pad capacitance is small but it is helpful to accurately model the measured data.

Out of these parameters, the series inductor $\left(L_{s}\right)$ affects the performance the most and changes in this value tend to move the modeled data away from the measured data very quickly.

In order to approximate the measured data correctly, we have to optimize the given component within tight limits of its tolerance values. For example, for a $56 \Omega$ resistor with nominal $( \pm 10 \%)$ tolerance, we have to use the optimization tolerance region from $50.4 \Omega$ to $61.6 \Omega$ to get good optimization result.

Figure 5.1 shows the percentage error of the modeled values of the resistors with respect to the nominal value. From the graph we see that the modeled values of the resistors are within $\pm 10 \%$ of the nominal data.

From the VNA measurements, we can see that there is moderate amount of variation among the values of resistors of the same value. Also, the resistors are in the specified tolerance band and calibration appears to work with an offset. If we could compensate for this offset, then we speculate that we could predict the exact value of the resistor.

The optimization does not work below a few MHz. One of the reasons for this is that calibration of the raw data does not work below a few MHz. Note that the calibration kit was not designed for low frequencies, below $0.5 \mathrm{GHz}$.

The LCR meter can measure a component from $20 \mathrm{~Hz}$ to $2 \mathrm{MHz}$ while the VNA can measure from $300 \mathrm{KHz}$ to $20 \mathrm{GHz}$. This provides us with an overlap region from $300 \mathrm{KHz}$ to $2 \mathrm{MHz}$ where we can compare the results from these two instruments. Table 5.2 shows the LCR meter data and the model values obtained 


\begin{tabular}{|c|c|c|}
\hline LCR meter data $(\Omega)$ & VNA data $(\Omega)$ & \% Difference \\
\hline \multicolumn{3}{|c|}{ Resistor $22 \Omega$} \\
\hline 21.95 & 23.59 & 7.47 \\
\hline 21.94 & 23.51 & 7.15 \\
\hline 21.96 & 24.00 & 9.28 \\
\hline 34.02 & 36.30 & 6.70 \\
\hline 34.03 & 35.49 & 4.29 \\
\hline 34.02 & 34.99 & 2.85 \\
\hline \multicolumn{3}{|c|}{ Resistor $56 \Omega$} \\
\hline 55.92 & 59.19 & 5.84 \\
\hline 55.93 & 59.19 & 5.82 \\
\hline 55.97 & 59.29 & 5.93 \\
\hline \multicolumn{3}{|c|}{ Resistor $75 \Omega$} \\
\hline 74.86 & 79.14 & 5.71 \\
\hline 74.91 & 78.60 & 4.92 \\
\hline 74.87 & 78.29 & 4.56 \\
\hline
\end{tabular}

Table 5.2: Comparison of resistor values obtained by using the LCR meter and those obtained using VNA measurements. LCR measurements were done from $300 \mathrm{KHz}$ to $2 \mathrm{MHz}$ and the VNA data in the same frequency range was compared. Average of these sets of measurements is shown.

from VNA measured data. The values that are shown in Table 5.2 differ from the values in Table 5.1. This is because the values in Table 5.2 are not optimized. This data was calculated from the S-parameters of the measured component after calibration was applied across the entire frequency range, i.e. from $300 \mathrm{kHz}$ to 10 GHz. We converted the S-parameters to Y-parameters and from the Y-parameters we converted it to B-parameter (part of $\mathrm{ABCD}$ matrix) which is equivalent to the impedance of the component.

For each value of resistor, three samples were measured on the LCR meter as well as the VNA. The average of these three values was plotted in Figure 5.2. The results from this graph gave us confidence since our measurements were comparable to the LCR meter results in the low frequency ranges. 


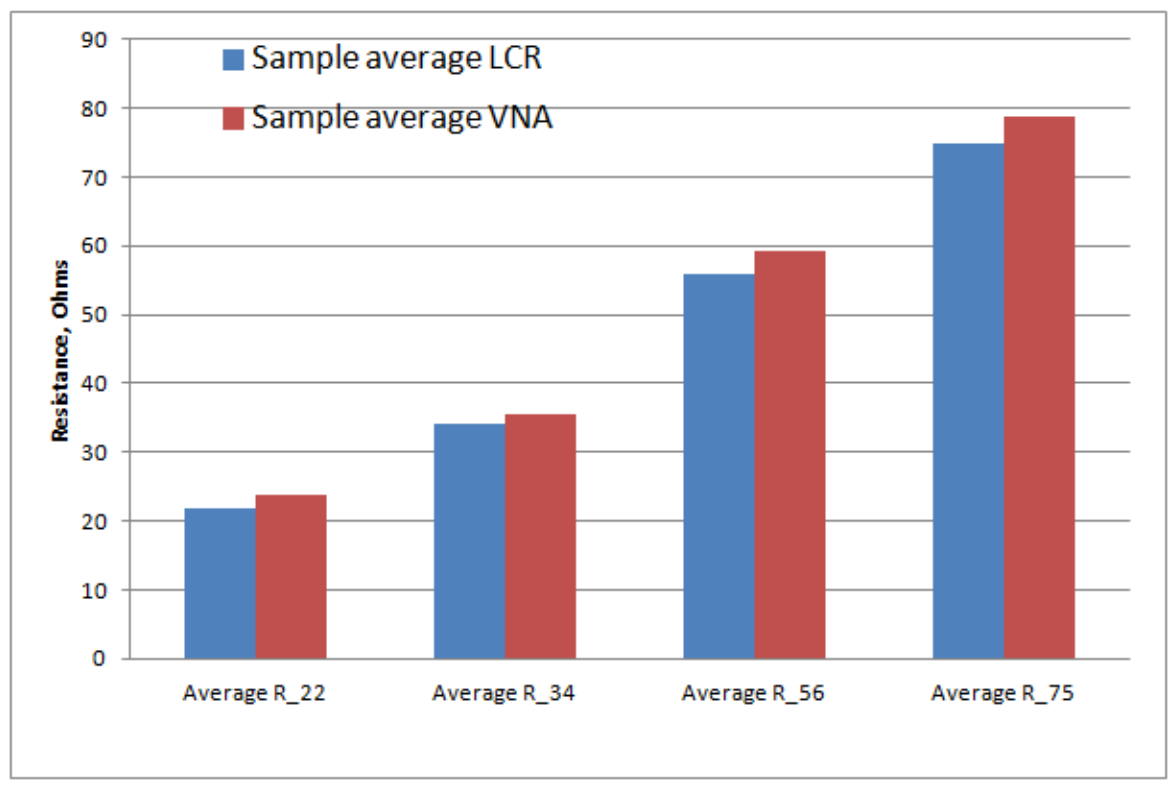

Figure 5.2: Comparison of average value of resistors measured using LCR and VNA.

From the results in Figure 5.2 and Table 5.2 we can see that the VNA measured results are within $\pm 10 \%$ of the LCR-measured value of the resistors and there is a constant offset in VNA-measured resistor values. We believe that this offset is due to non-ideal microstrip transmission lines and their effect on calibration. Ideally the lines were supposed to be $50 \Omega$, but after manufacturing we found that the lines have an impedance between $47.3 \Omega$ and $49.5 \Omega$ according to the measurement of the short and open on TDR.

\subsection{Inductor Results}

Table 5.3 shows values of model parameters for SMD inductors after optimization was performed in ADS.

From the Table 5.3 we can see that pad capacitances for inductors of different values are very close to each other and also very small. 


\begin{tabular}{|l|l|l|l|l|}
\hline Inductor(nH) & $\mathrm{Rs}(\mathrm{ohms})$ & $\mathrm{Cp}(\mathrm{pF})$ & $\mathrm{Cpad}(\mathrm{pF})$ & Cpad2(pF) \\
\hline \multicolumn{5}{|c|}{ Inductor $22 \mu \mathrm{H}$} \\
\hline 24779 & 0.21 & 1.15 & 0.08356 & 0.096 \\
\hline 24279 & 0.23 & 1.17 & 0.08355 & 0.096 \\
\hline 24046 & 0.20 & 1.18 & 0.08357 & 0.095 \\
\hline \multicolumn{5}{|c|}{ Inductor 2.2 $\mathrm{nH}$} \\
\hline 2.7 & 0.21 & 0.47 & 0.0834 & 0.096 \\
\hline 2.5 & 0.54 & 0.43 & 0.0601 & 0.059 \\
\hline 2.7 & 0.89 & 0.44 & 0.0835 & 0.096 \\
\hline \multicolumn{5}{|c|}{ Inductor $22 \mathrm{nH}$} \\
\hline 26.39 & 1.09 & 0.1490 & 0.0835 & 0.0964 \\
\hline 25.30 & 1.64 & 0.1005 & 0.0235 & 0.0134 \\
\hline 24.20 & 1.68 & 0.1252 & 0.0235 & 0.0134 \\
\hline
\end{tabular}

Table 5.3: Inductor model parameters for a given nominal component value. Measurement done on VNA.

The value of shunt capacitance Cp affects the inductor model to a great extent. If we loosen the optimization criteria (the limit of $\mathrm{Cp}$ ) then the resonance dip in S12 or S21 disappears as its position increases in frequency. See Figure 4.8 for an illustration.

Figure 5.3 shows the percentage error of the modeled values of the inductors with respect to the nominal value. From the graph we see that the modeled values of the inductors have a high percentage of relative error. This is because of the measurement problem that we faced. The $2.2 \mathrm{nH}$ and $22 \mathrm{nH}$ inductors had surface contacts so it was difficult to solder these components and take measurements. On the other hand percentage error for $22 \mu \mathrm{H}$ is relatively low since it had contact pads on the side which made it easier to solder as well as for taking measurements using the LCR meter.

Table 5.4 shows the results of measurement of $22 \mu \mathrm{H}$, inductor using the VNA and LCR meter. The other two inductor measurements were not trusted. The inductor values were calculated in a similar way as discussed in the previous section 


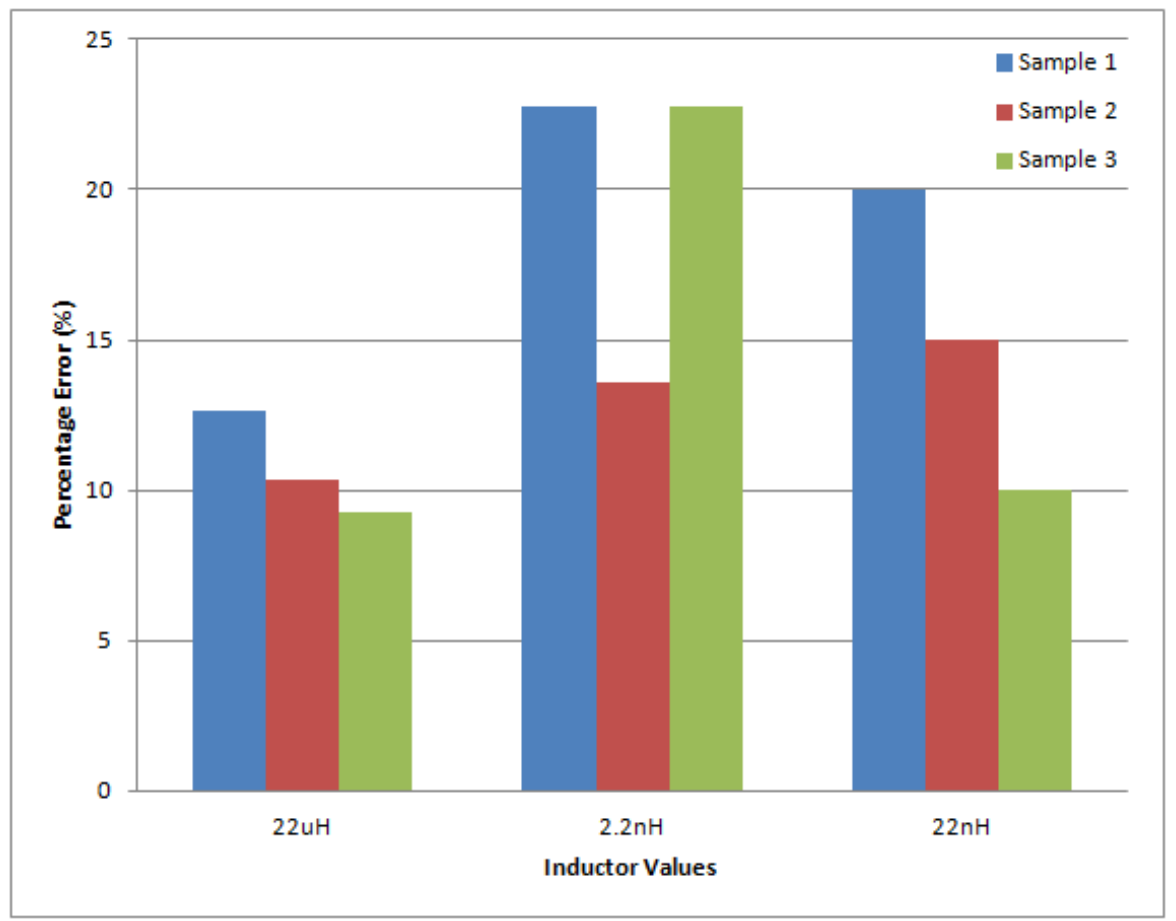

Figure 5.3: Inductor percentage error with respect to nominal value for three samples.

for resistors.

Three samples of $22 \mu \mathrm{H}$ inductor were measured on the LCR meter as well as the VNA. The VNA data shown in Table 5.4 is calibrated but not optimized. We suspect that the results are same in all the three cases due to larger size of the inductor. The size of the $22 \mu \mathrm{H}$ inductor was 0805. The average of these three values was plotted in Figure 5.4. The results from this graph gave us confidence since our measurements were comparable to the LCR meter results in the low frequency ranges. We were able to measure only this inductor on the LCR meter since it had contact pads on the sides. 


\begin{tabular}{|c|c|}
\hline $\mathrm{LCR} \mathrm{r}$ & VNA data $(\mu \mathrm{H})$ \\
\hline \multicolumn{2}{|c|}{ Inductor $22 \mu \mathrm{H}$} \\
\hline 23.73 & 22.77 \\
\hline 24.07 & 22.77 \\
\hline 23.55 & 22.77 \\
\hline
\end{tabular}

Table 5.4: Comparison of inductor values obtained by using the LCR meter and those obtained using VNA measurements. LCR measurements were done from 300 $\mathrm{KHz}$ to $2 \mathrm{MHz}$ and the VNA was measured at these same frequencies. Average of these sets of measurements is shown.

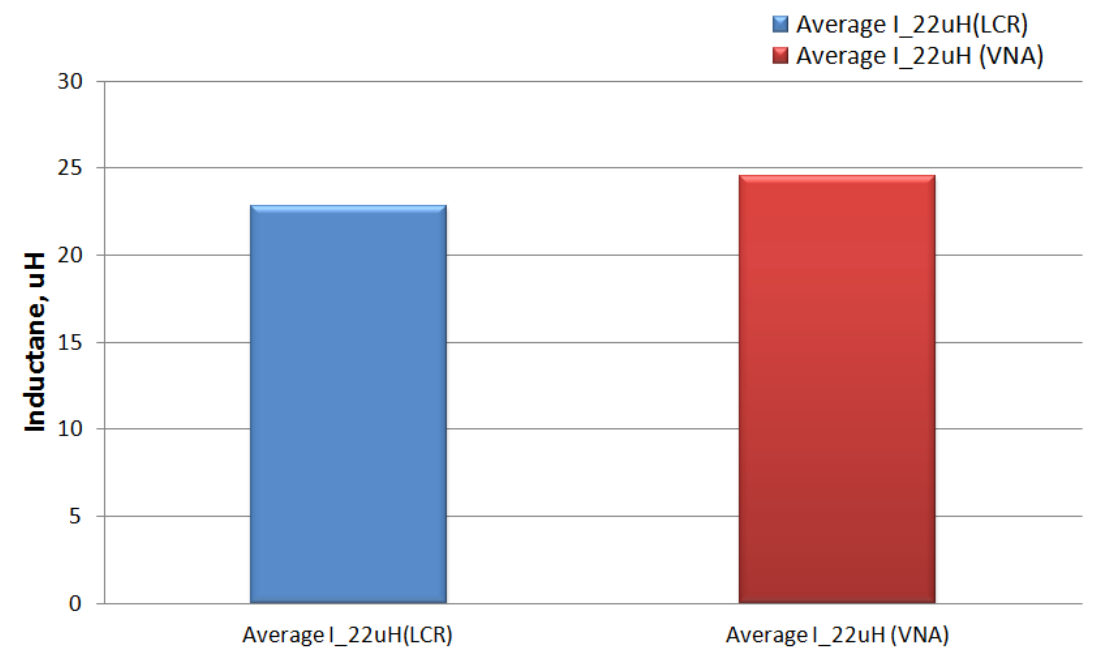

Figure 5.4: Comparison of average value of $22 \mu \mathrm{H}$ inductor measured using LCR and VNA. 


\begin{tabular}{|l|l|l|l|l|}
\hline Capacitor(pF) & Rs(ohms) & Ls(nH) & Cpad1(pF) & Cpad2(pF) \\
\hline \multicolumn{5}{|c|}{ Capacitor 1.5 pF } \\
\hline 1.42 & 0.760 & 1.36 & 0.0832 & 0.0964 \\
\hline 1.41 & 0.989 & 1.37 & 0.0832 & 0.0964 \\
\hline 1.41 & 0.989 & 1.34 & 0.0773 & 0.0916 \\
\hline \multicolumn{5}{|c|}{ Capacitor 3.3 pF } \\
\hline 3.16 & 0.22 & 1.22 & 0.0832 & 0.0965 \\
\hline 3.22 & 0.21 & 1.37 & 0.0833 & 0.0964 \\
\hline 3.20 & 0.15 & 1.43 & 0.0821 & 0.0965 \\
\hline \multicolumn{5}{|c|}{ Capacitor $15 \mathrm{pF}$} \\
\hline 13.66 & 0.27 & 1.42 & 0.0832 & 0.0964 \\
\hline 14.25 & 0.98 & 1.00 & 0.0230 & 0.0964 \\
\hline 14.02 & 0.34 & 1.71 & 0.0832 & 0.0964 \\
\hline \multicolumn{5}{|c|}{ Capacitor 33 pF } \\
\hline 30.47 & 0.35 & 1.49 & 0.0802 & 0.0964 \\
\hline 29.57 & 0.15 & 1.54 & 0.0833 & 0.0936 \\
\hline 29.80 & 0.15 & 1.55 & 0.0791 & 0.0964 \\
\hline
\end{tabular}

Table 5.5: Capacitor model parameters for a given nominal component value. Measurement done on VNA.

\subsection{Capacitor Results}

Table 5.5 shows the results of parasitic components of the capacitor that were calculated by the optimized model in ADS. We were able to model the same component with a very simple model yet we were able to get the results comparable to the measured data.

We can also observe that the pad capacitances are almost the same and have a small value across the complete range of capacitors measured. In the case of the capacitor, the series inductance plays a major role in determining the resonant frequency. In order to get the model closer to the measured data this series inductance was subjected to tight bounds of optimization tolerance.

Figure 5.5 shows the percentage error of the modeled values of the capacitors with respect to the nominal value. From the graph we see that the modeled values 


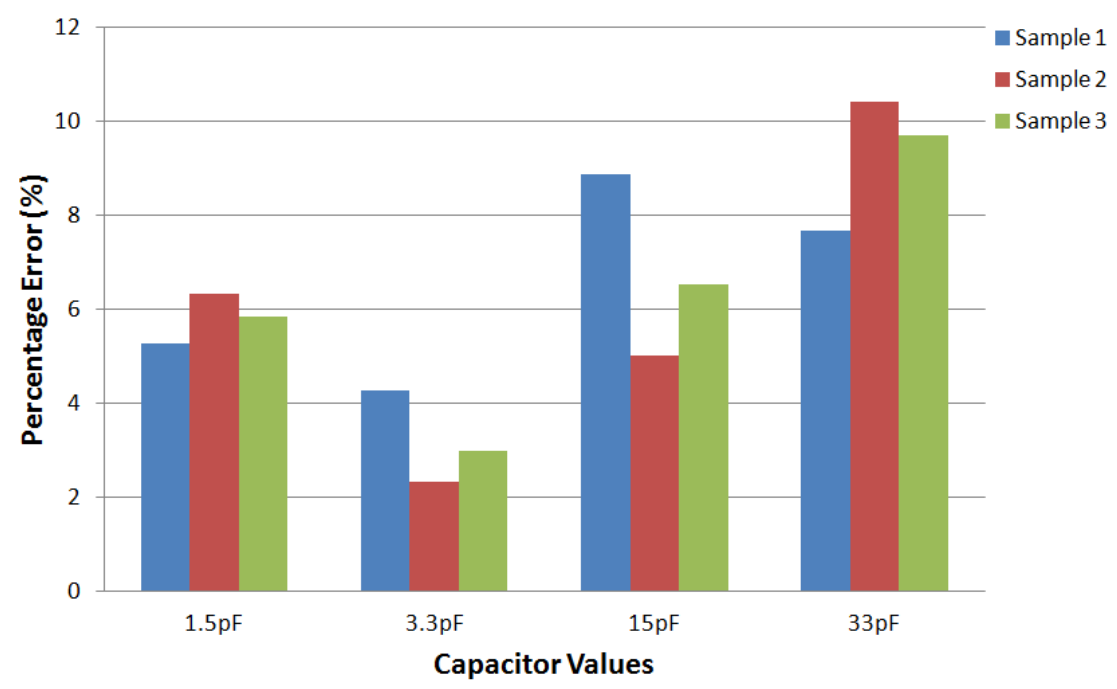

Figure 5.5: Capacitor percentage error with respect to nominal value for three samples.

of the capacitors vary more than resistor but less than inductor. For the lower values of capacitor our model gives smaller error but all of the values were within $\pm 10 \%$ of the nominal value. As the value of the capacitor increases the VNA optimized data is moving away from the nominal value and the final value is closer to the tolerance band.

Table 5.6 shows the VNA measured data is comparable to the measured data using the LCR meter. We also observe that the VNA data is within the tolerance range of the nominal value specified by the manufacturer. The offset in the measurement is due to the impedance mismatch of the transmission lines and calibration artifact as explained in the previous section on resistors.

For each value of capacitor, three samples were measured on the LCR meter as well as the VNA. The average of these three values was plotted in Figure 5.6. The results from this graph gave us confidence since our measurements were comparable to the LCR meter results in the low frequency ranges. 


\begin{tabular}{|c|c|c|}
\hline LCR meter data(pF) & VNA data $(\mathrm{pF})$ & \% Difference \\
\hline \multicolumn{3}{|c|}{ Capacitor $1.5 \mathrm{pF}$} \\
\hline 1.49 & 1.42 & 4.69 \\
\hline 1.48 & 1.40 & 5.40 \\
\hline 1.48 & 1.39 & 6.08 \\
\hline \multicolumn{3}{|c|}{ Capacitor $3.3 \mathrm{pF}$} \\
\hline 3.38 & 3.15 & 6.80 \\
\hline 3.36 & 3.20 & 4.76 \\
\hline 3.35 & 3.22 & 3.88 \\
\hline \multicolumn{3}{|c|}{ Capacitor $15 \mathrm{pF}$} \\
\hline 14.82 & 14.25 & 3.84 \\
\hline 14.59 & 14.28 & 2.12 \\
\hline 15.20 & 14.24 & 6.31 \\
\hline \multicolumn{3}{|c|}{ Capacitor $33 \mathrm{pF}$} \\
\hline 32.95 & 31.35 & 4.85 \\
\hline 32.68 & 31.34 & 4.10 \\
\hline 32.70 & 30.93 & 5.41 \\
\hline \multicolumn{3}{|c|}{} \\
\hline
\end{tabular}

Table 5.6: Comparison of capacitor values obtained by using LCR meter and those obtained using VNA measurements. LCR measurements were done from $300 \mathrm{KHz}$ to $2 \mathrm{MHz}$ and the VNA was measured at these same frequencies. Average of these sets of measurements is shown.

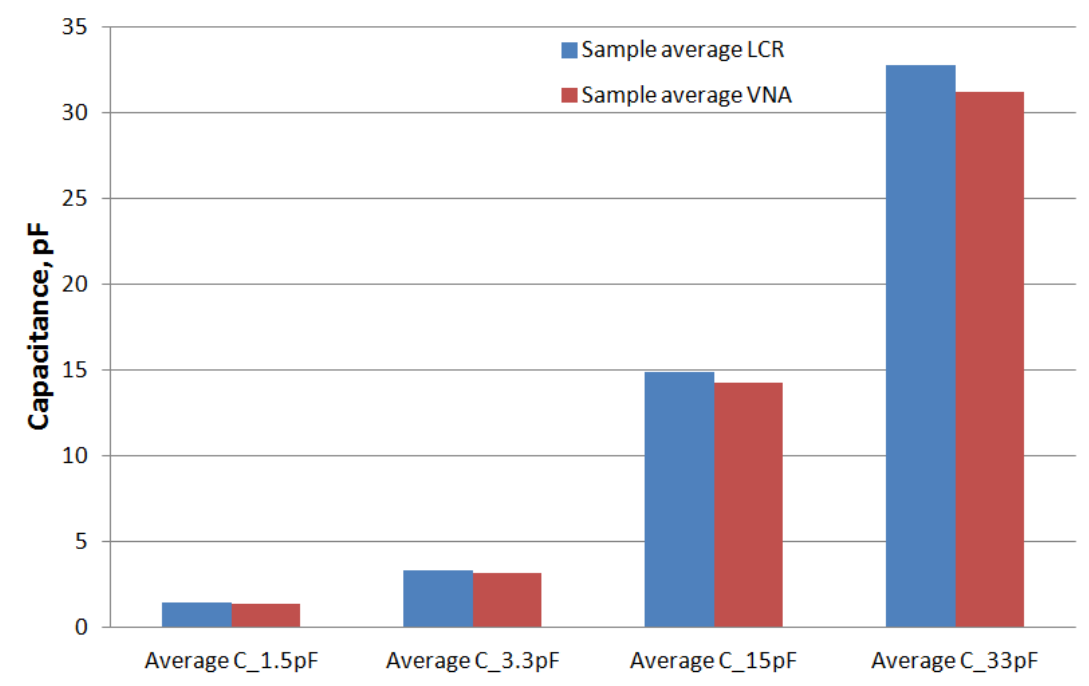

Figure 5.6: Comparison of average value of capacitors measured using LCR and VNA. 


\section{Chapter 6}

\section{Conclusion and Future work}

\subsection{Conclusions}

The following are some of the major contributions and conclusions of this study:

- Inexpensive and simple fixtures based on microstrip lines were developed on FR4 substrates. These fixtures were used to characterize the passive surface mount devices which gave us results that are comparable to the actual data provided by the component manufacturers.

- The calibration kit that was developed for the TRL calibration algorithm works reasonably well up to roughly $4 \mathrm{GHz}$. From the experiments that were carried out with it, it can be concluded that shorter lines can be used for higher frequencies as compared to the longer lines.

- Simple models that were developed for the extraction of the passive SMD components on FR4 microstrip transmission line environment matched the measurements well and were sufficiently accurate up to $4 \mathrm{GHz}$.

- VNA results were compared with LCR meter results and general agreement was observed but some discrepancies were observed that we attribute to nonideal characteristic impedance of transmision lines and the subsequent errors in TRL calibration.

During this work we also accomplished the following: 
- Overall, a well-defined procedure for the measurements, calibration and extraction of parameters of passive devices was developed.

- Simple equivalent circuit models were implemented in ADS for SMD resistors, inductors and capacitors.

- Procedures for designing layout and having it manufactured at PCB foundry were developed and documented.

\subsection{Future work}

- The inductors that were used had the pads only on the bottom surface so it was difficult to measure them on the LCR meter. Use inductors that have accessible pad contacts so that we can measure these inductors using the LCR meter. This would make inductor characterization easier and more reliable.

- Investigate other calibration algorithms such as SOLT, LRM etc.

- Understand the effect of these models by changing the substrate from FR4 to some other substrate like duriod.

- Using the PCB manufacturer's data, generate a microstrip transmission line environment which is exactly 50 Ohm.

- Model the SMD components in a coplanar waveguide design environment. 


\section{References}

[1] AgilentTechnologies. Agilent an 154 s-parameter design application note. Technical report, Agilent Technologies, 2005.

[2] AgilentTechnologies. Agilent network analysis applying the 8510 trl calibration for non-coaxial measurements product note 8510-8a. Technical report, Agilent Technologies, 2005.

[3] AgilentTechnologies. Network analyzer measurements: Filter and amplifier examples. Agilent AN 1287-4, 2006.

[4] AgilentTechnologies. Agilent impedance measurement handbook. A guide to measurement technology and techniques 4th Edition, Chapter 1 and 5, 2009.

[5] AgilentTechnologies. Ads 2011 help. Technical report, Agilent Technologies, 2011.

[6] E. Michielssen F. Ulaby and U. Ravaioli. Fundamentals of Applied Electromagnetics, 6th ed. Prentice Hall, 2010.

[7] Eric L. Holzman. Wideband measurement of the dielectric constant of an fr-4 substrate using a parallel-coupled microstrip resonator. In IEEE Transactions on Microwave Theory and Techniques, vol.54, no. 7, pp. 3127-3130, July 2006., July 2006.

[8] David M. Pozar. Microwave Engineering, 3rd Edition. Wiley, 2005. 
[9] J. Capwell V. Cojocaru, D. Markell. Accurate simulation of rf designs requires consistent modeling techniques. In High Frequency Electronics, Summit Technical Media, LLC, September 2002.

[10] Scott A. Wartenberg. RF measurements of Die and Packages. Artech House, 2002.

[11] Wikipedia. Resistors, capacitors and inductors. Wikipedia, 2012. 
Appendix A

Plots

A.1 Resistor plots

A.2 Capacitor plots

A.3 Inductor plots 


\begin{tabular}{|cc|}
\hline$d B(S(1,1))$ & $=-$ \\
$d B(S 11 m)$ & $=-$ \\
$d B($ resistor_34_Vishay_model..S(1,1)) & \\
\hline
\end{tabular}
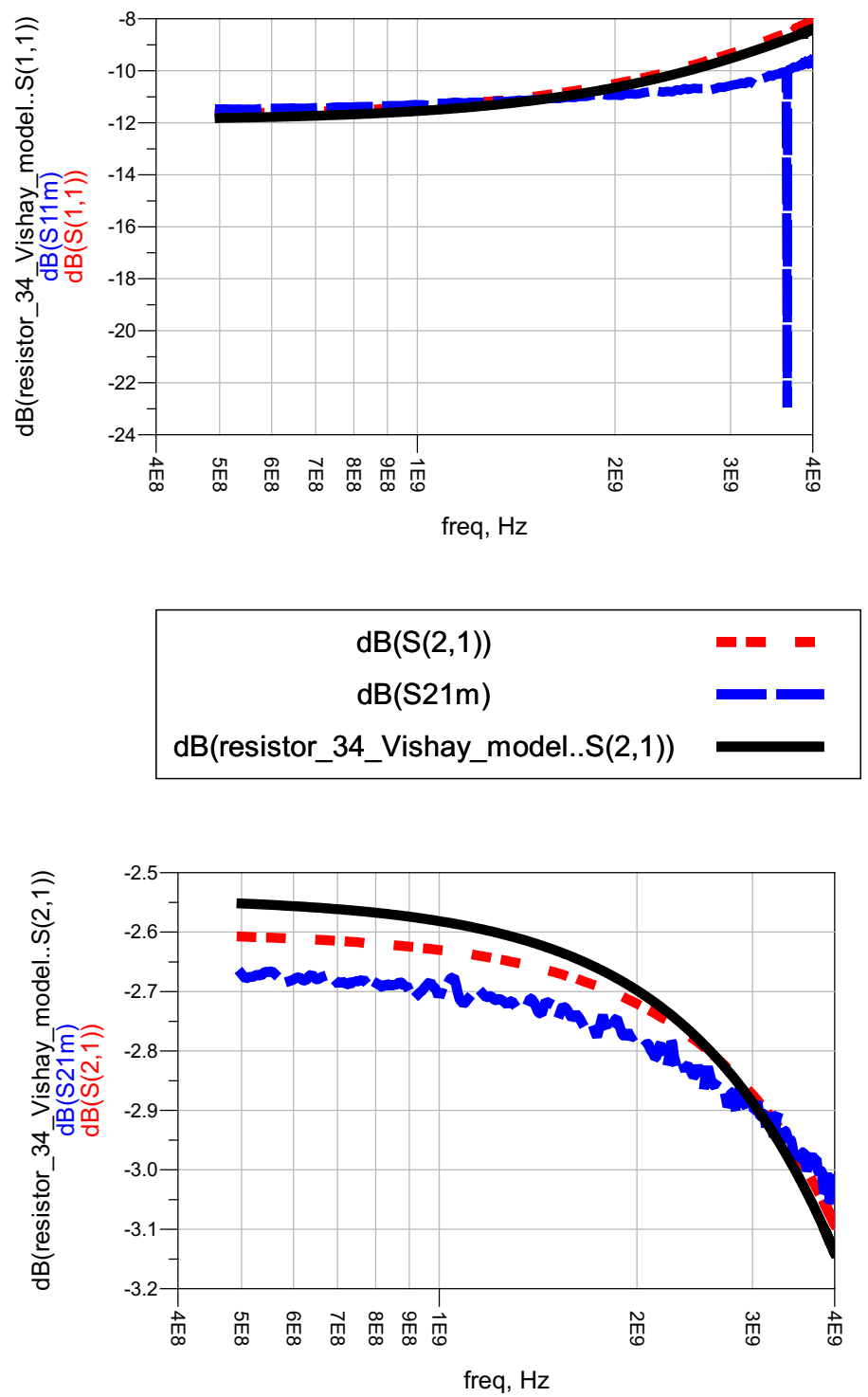

Figure A.1: Comparison of extracted model (red), measured component (blue) and nominal model (black) values for S11 and S21 of $34 \Omega$ resistor. 


\begin{tabular}{|cc|}
\hline $\mathrm{dB}(\mathrm{S}(1,1))$ & $=-$ \\
$\mathrm{dB}(\mathrm{S} 11 \mathrm{~m})$ & - \\
$\mathrm{dB}$ (resistor_56_Vishay_model..S(1,1)) & \\
\hline
\end{tabular}
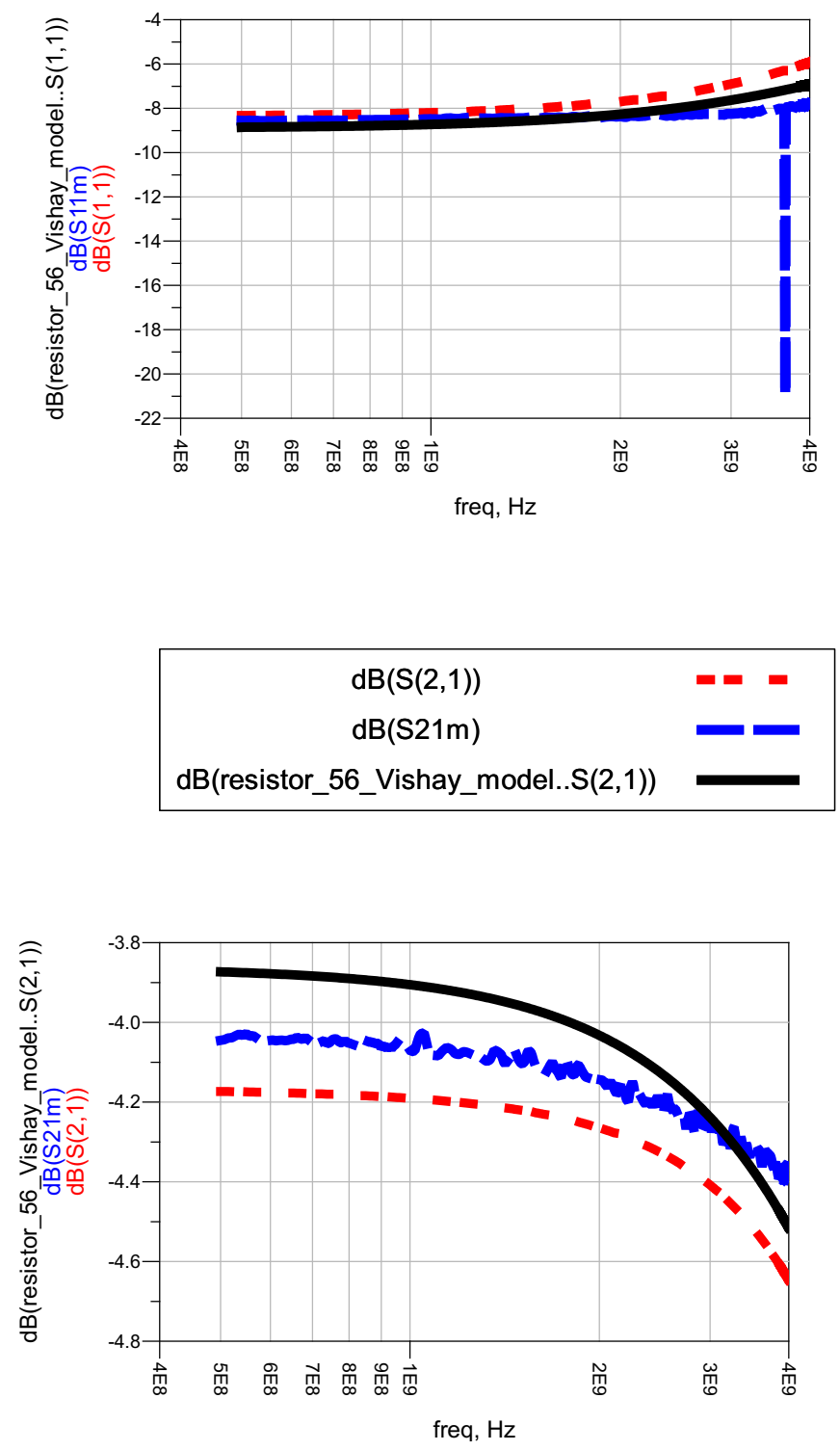

Figure A.2: Comparison of extracted model (red), measured component (blue) and nominal model (black) values for S11 and S21 of $56 \Omega$ resistor. 

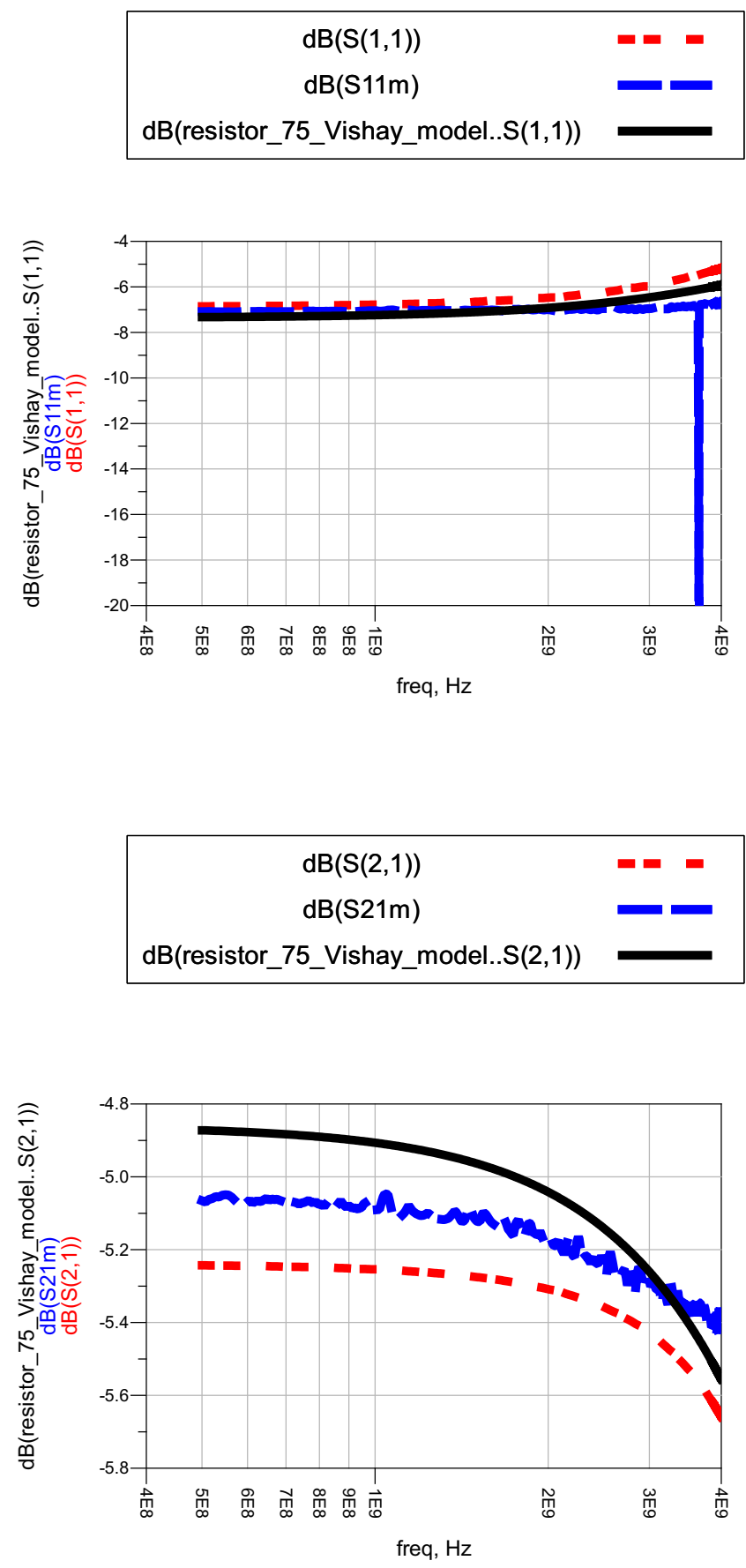

Figure A.3: Comparison of extracted model (red), measured component (blue) and nominal model (black) values for S11 and S21 of $75 \Omega$ resistor. 


\begin{tabular}{|c|c|}
\hline $\mathrm{dB}(\mathrm{S}(1,1))$ & $=-$ \\
\hline $\mathrm{dB}(\mathrm{S} 11 \mathrm{~m})$ & \\
\hline $\mathrm{dB}($ Cap_1_5pF_Murata..S(1,1)) & \\
\hline
\end{tabular}
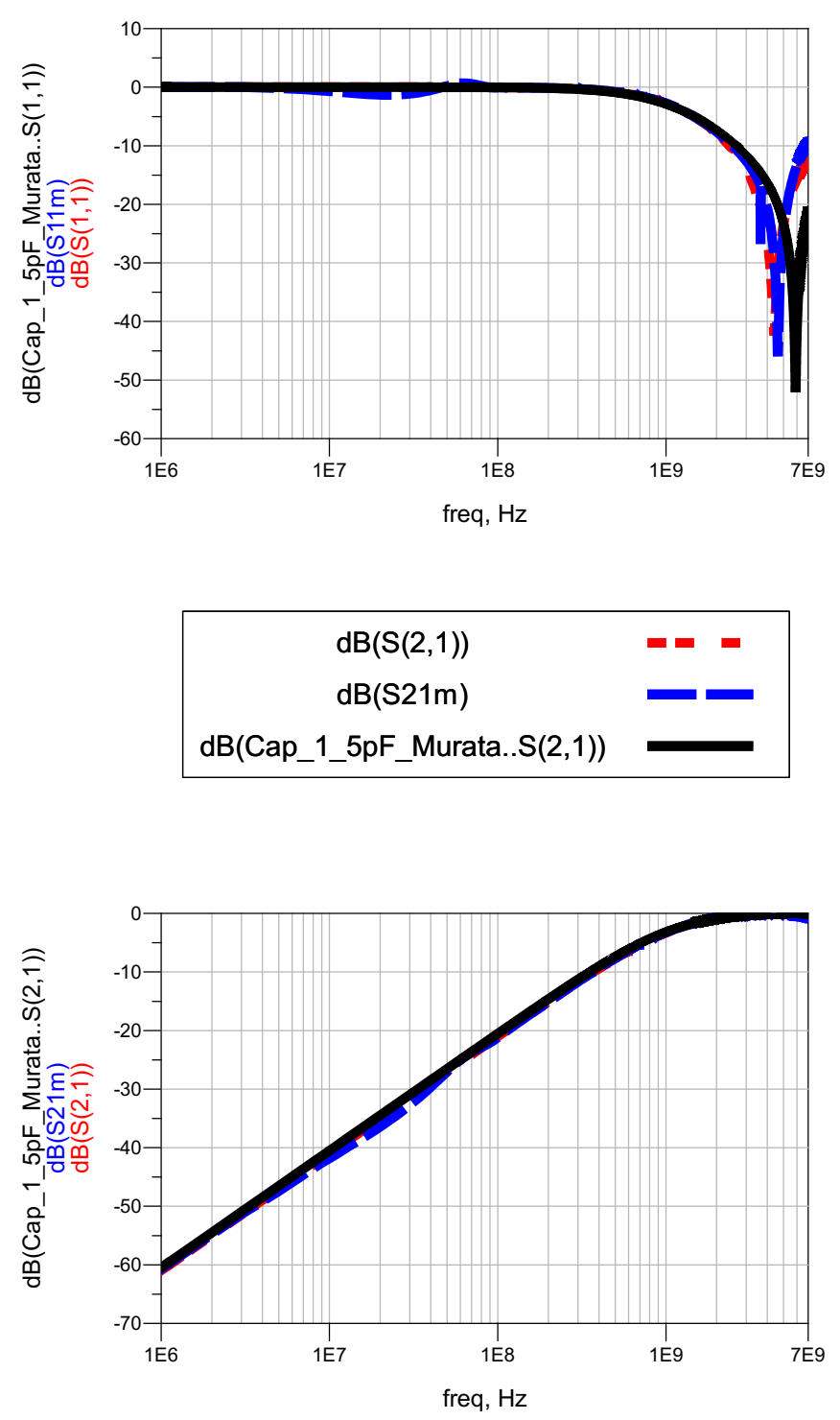

Figure A.4: Comparison of extracted model (red), measured component (blue) and nominal model (black) values for S11 and S21 of $1.5 \mathrm{pF}$ capacitor. 

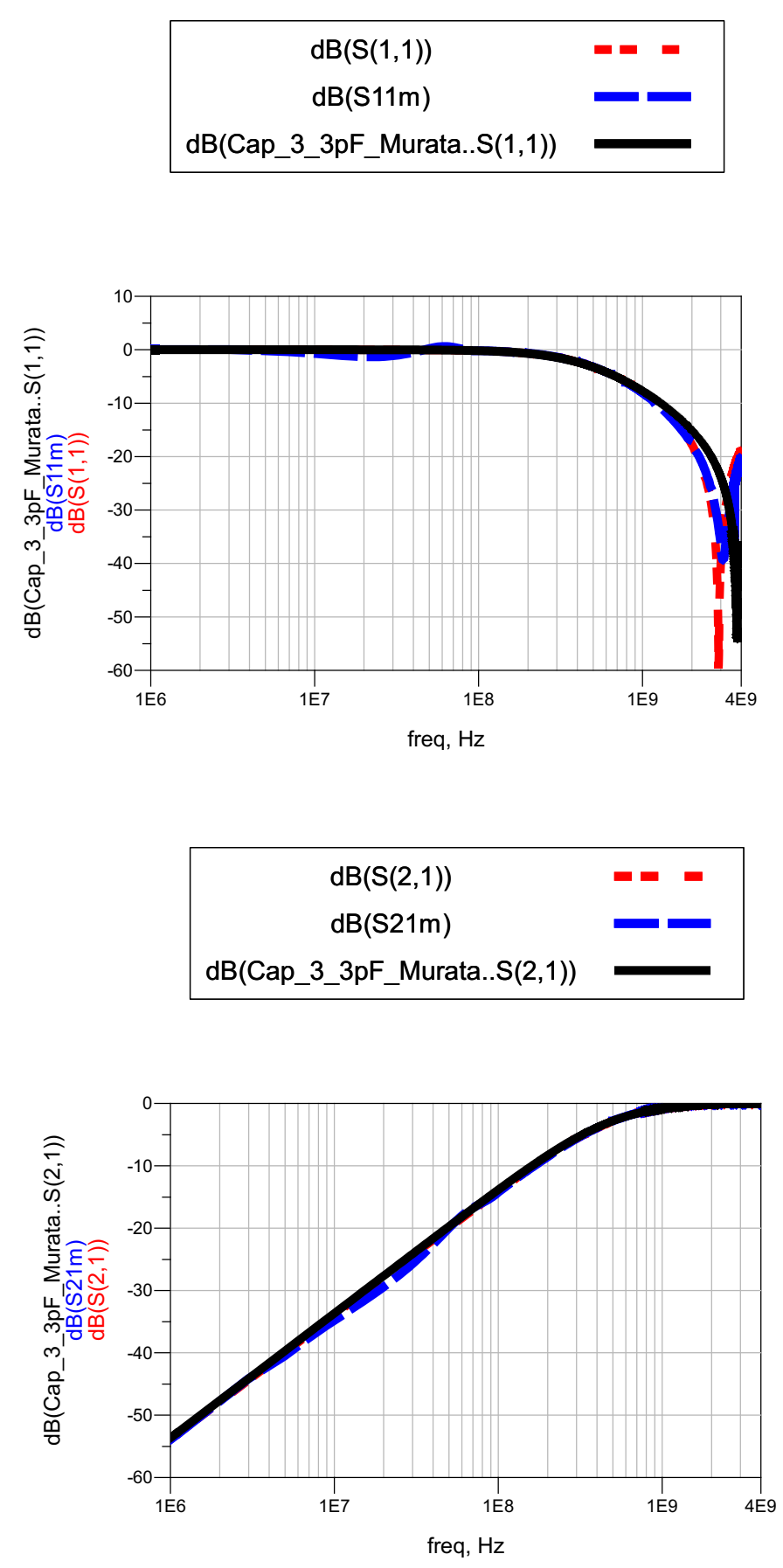

Figure A.5: Comparison of extracted model (red), measured component (blue) and nominal model (black) values for S11 and S21 of $3.3 \mathrm{pF}$ capacitor. 

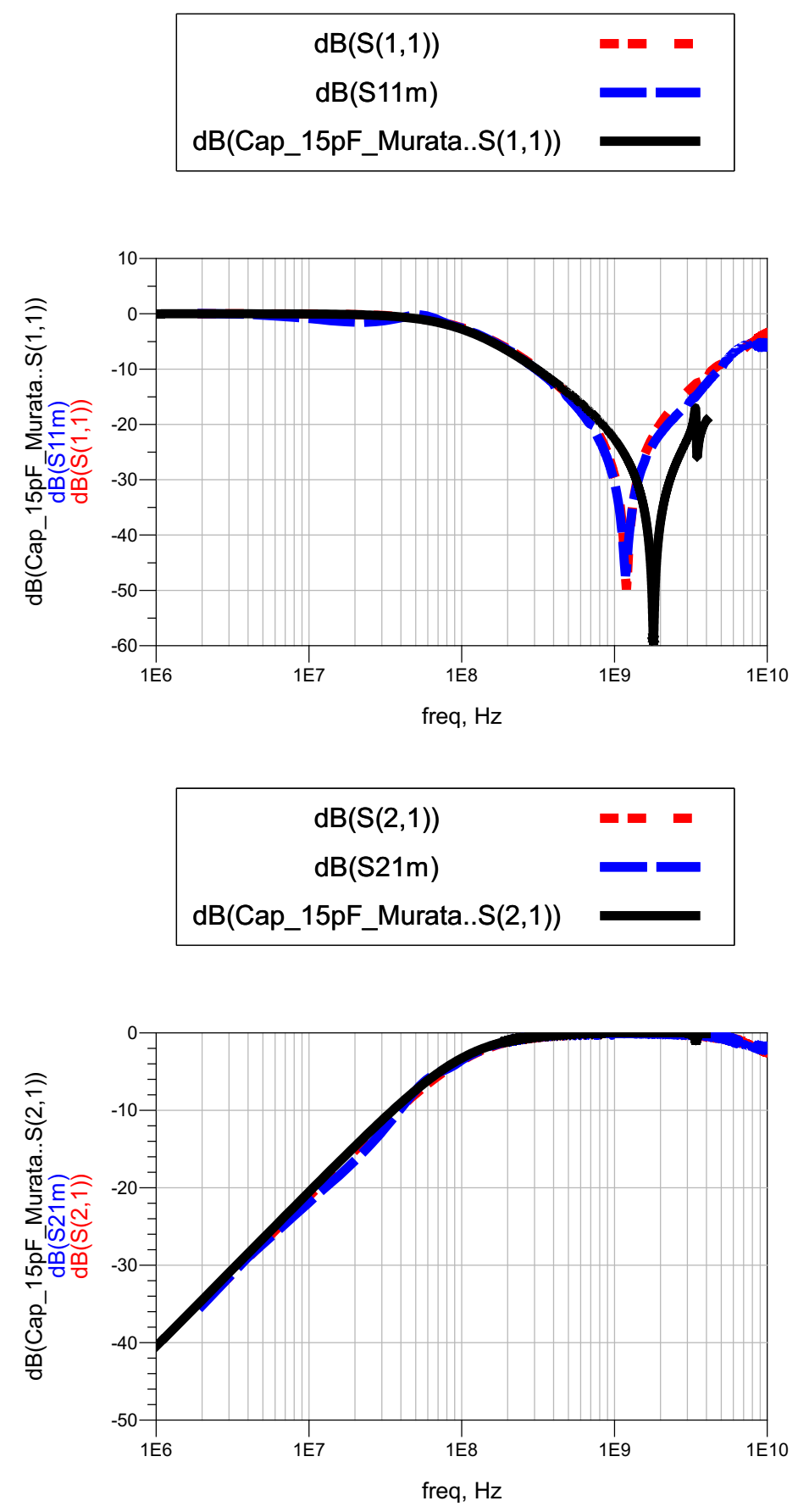

Figure A.6: Comparison of extracted model (red), measured component (blue) and nominal model (black) values for S11 and S21 of $15 \mathrm{pF}$ capacitor. 


$$
\begin{aligned}
& \mathrm{dB}(\mathrm{S}(1,1))= \\
& \mathrm{dB}(\mathrm{S} 11 \mathrm{~m})=
\end{aligned}
$$
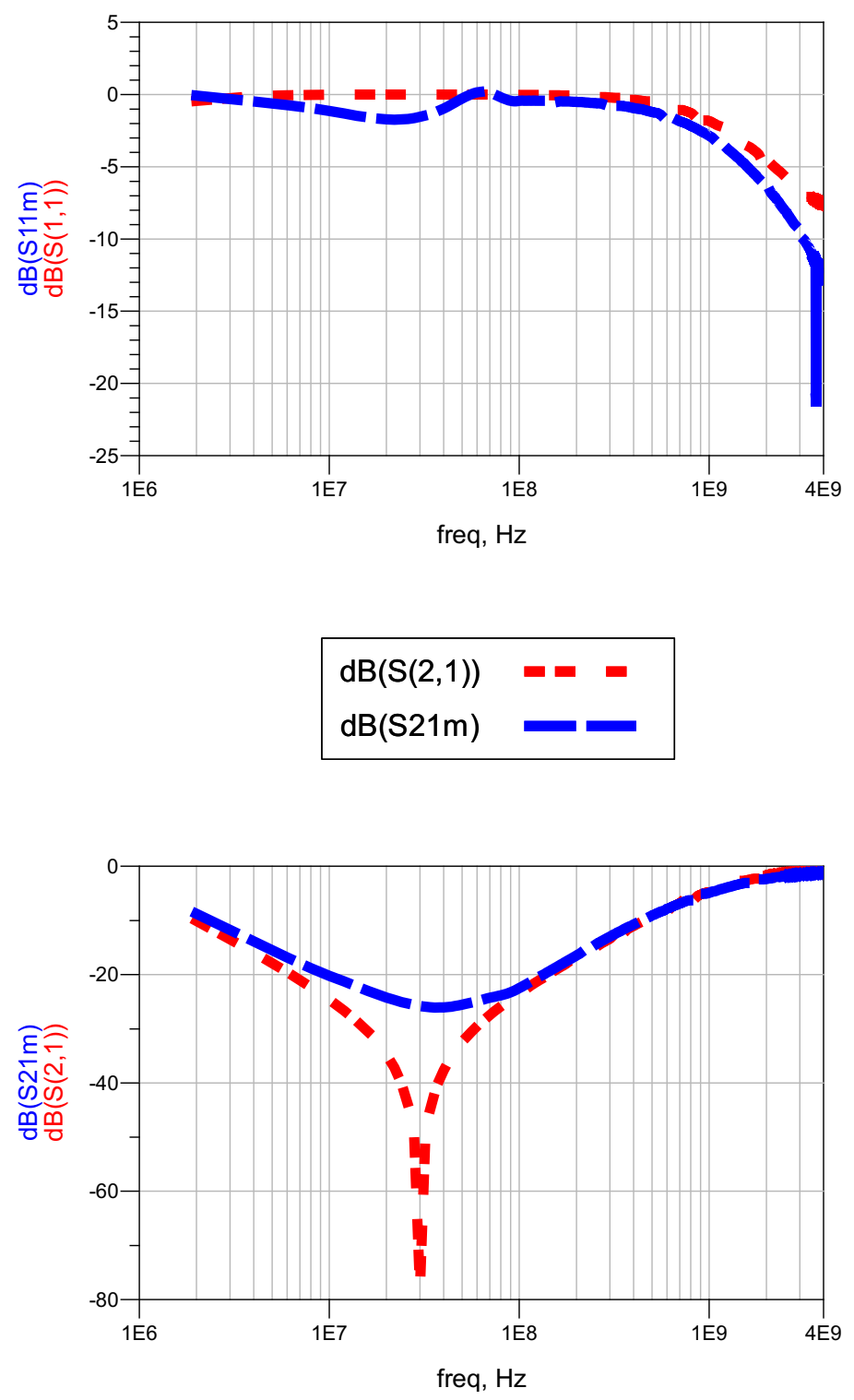

Figure A.7: Comparison of extracted model (red), measured component (blue) and nominal model (black) values for S11 and S21 of $22 \mu \mathrm{H}$ inductor. 

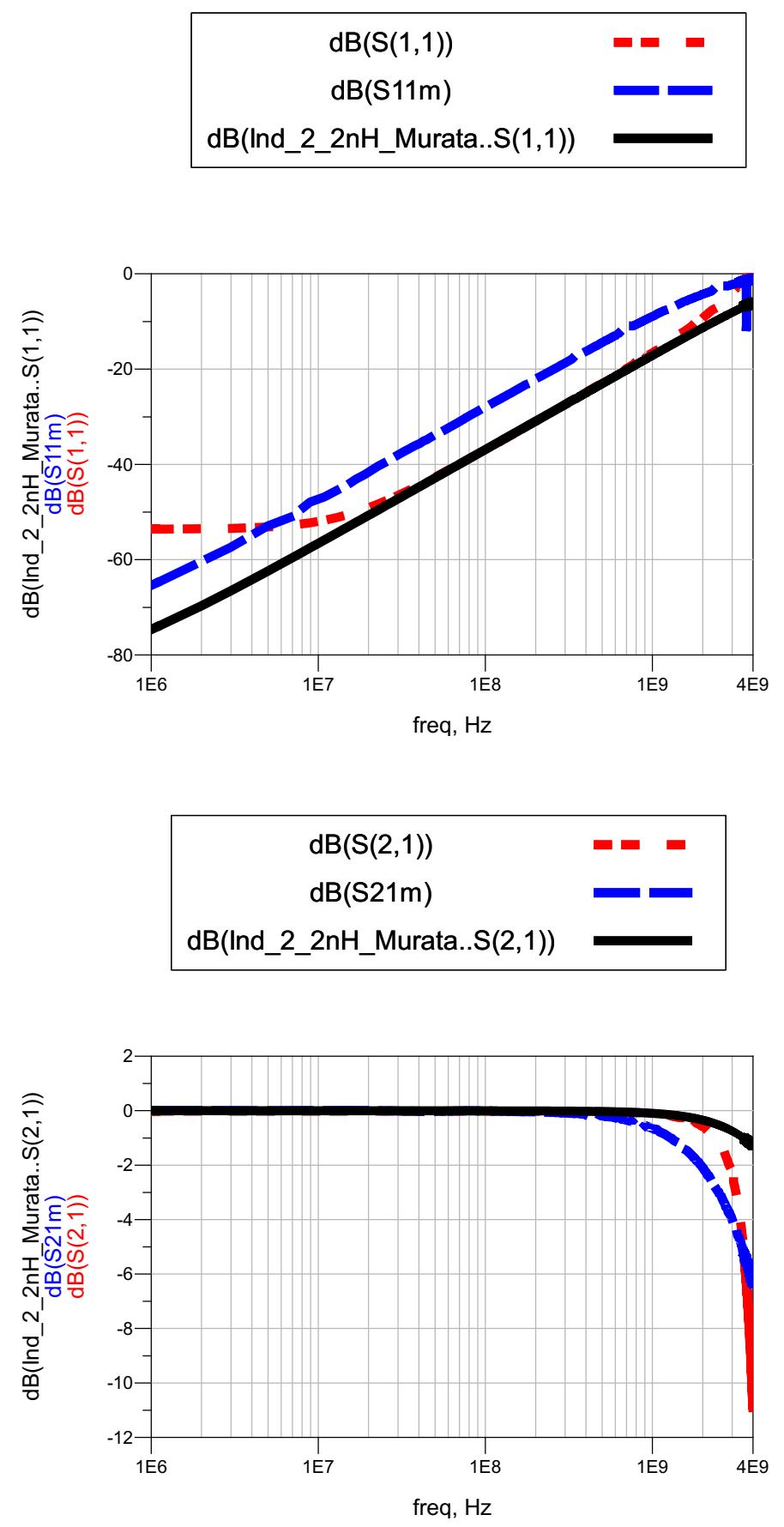

Figure A.8: Comparison of extracted model (red), measured component (blue) and nominal model (black) values for S11 and S21 of $2.2 \mathrm{nH}$ inductor. 